\title{
SOME ASYMPTOTIC FIXED POINT THEOREMS
}

\author{
BY
}

ROGER D. NUSSBAUM( $\left.{ }^{1}\right)$

\begin{abstract}
By an asymptotic fixed point theorem we mean a theorem in functional analysis in which the existence of fixed points of a map $f$ is established with the aid of assumptions on the iterates $f^{n}$ of $f$. We prove below some new theorems of this type, and we obtain as corollaries results of F. E. Browder, G. Darbo, R. L. Frum-Ketkov, W. A. Horn and others. We also state a number of conjectures about fixed point theorems at the end of the paper.
\end{abstract}

Our interest in the results here is two-fold. First, asymptotic fixed point theorems have proved useful in the theory of ordinary and functional differential equations (see [17], [18], [19] and [34]), and in fact we hope to indicate in a future paper some applications of our results to functional differential equations of neutral type (see [11] or [15]). Second, and perhaps more relevant to our immediate line of development, asymptotic fixed point theorems provide a framework for unifying and generalizing many of the known fixed point theorems of functional analysis.

The immediate impetus for this paper comes from the following theorem, which was claimed by R. L. Frum-Ketkov in [13]:

Theorem. Let $B$ be a closed ball in a real Banach space $X$ and $f: B \rightarrow B$ a continuous map. Assume that there exist a constant $-k<1$ and a compact set $K \subset X$ such that for all $x \in B, d(f(x), K) \leq k d(x, K)$, where $d(y, K)$ denotes the distance from a point $y$ to $K$. Then $f$ bas a fixed point.

As has been remarked in [25] and [27], Frum-Ketkov's proof seems to be in error. Specifically, one can construct a function $f$ defined on the unit ball in Euclidean 2-space which contradicts Frum-Ketkov's assertion that the numbers $c(f, T)$ considered in [13] stabilize mod 2. A correct proof of Frum-Ketkov's theorem for the case that the Banach space is essentially a $\pi_{1}$-space (e.g., a Hilbert space or an $L^{p}$ space, $\left.1 \leq p<\infty\right)$ was given in [25] and [27]. Subsequently, F. E. Browder generalized Frum-Ketkov's theorem (see [7, Theorem 16.3]), but only for Hilbert space. Browder's proof makes essential use of geometrical properties of Hilbert space and does not generalize directly to Banach spaces.

Presented to the Society, March 27, 1971; received by the editors September 4, 1970. AMS 1969 subject classifications. Primary 4785, 4780; Secondary 5485.

Key words and phrases. Fixed points, asymptotic fixed point theorems, fixed point index, generalized Lefschetz number, measure of noncompactness, compact map, $k-$ setcontraction.

(1) Partially supported by NSF GP-20228. 
We shall obtain Frum-Ketkov's theorem from a very general result (Theorem 1 below). One might think that if one were only interested in Frum-Ketkov's theorem, an easier proof would be possible. We know of no proof of Frum-Ketkov's theorem, however, which does not essentially use all the ideas of Theorem 1.

1. We begin our work by recalling a geometrical result which will be of crucial importance here. Variants of Lemma 1 play a key role in the development of the fixed point index for $k$-set-contractions in [24], [26], [28] and also form the basis for the geometrical approach to the classical fixed point index in [27]. Lemma 1 can be viewed as a generalization of a theorem of Dugundji [12].

Lemma 1 (Nussbaum [26]). Let $C$ and D be closed subsets of a Banach space $X, C \supset D$. Assume that $C=\bigcup_{j=1}^{n} C_{j}$ and $D=\bigcup_{j=1}^{n} D_{j}$, where $C_{j}$ and $D_{j}$ are closed, convex subsets of $X$ and $C_{j} \supset D_{j}$ for $1 \leq j \leq n$. For each subset $J \subset\{1,2$, $\cdots, n\}$ assume that $C_{J}=\bigcap_{j \in J} C_{j}$ is nonempty if and only if $D_{J}=\bigcap_{j \in J} D_{j}$ is nonempty. Then there exists a retraction $R$ of $C$ onto $D$ such that $R\left(C_{j}\right) \subset C_{j}$ for $1 \leq j \leq n$.

Notice that Lemma 1 implies in particular that $D$ is a deformation retract of $C$ by the deformation retraction $H: C \times[0,1] \rightarrow C$ given by $H(x, t)=t R(x)+$ $(1-t) x$.

As an immediate corollary of Lemma 1, we have the following simple result, which we shall need later.

Lemma 2. Let $C$ be a closed subset of a Banach space $X$ and assume that $C=\bigcup_{j=1}^{n} C_{j}, C_{j}$ closed and convex. Let $K$ be a closed convex set and assume that for each $J \subset\{1,2, \cdots, n\}$ if $C_{J}=\bigcap_{j \in J} C_{j}$ is nonempty, then $C_{J} \cap K$ is nonempty. Let $C^{\prime}=C \cup K$. Then $C^{\prime}$ is contractible in itself to a point, i.e., there exists a continuous map $H: C^{\prime} \times[0,1] \rightarrow C^{\prime}$ sucb that $H(x, 0)=x$ for all $x \in C^{\prime}$ and $H(x, 1)=x_{0}$, where $x_{0}$ is a given point in $C^{\prime}$, independent of $x$.

Proof. For each subset $J \subset\{1,2, \cdots, n\}$ such that $\bigcap_{j \in J} C_{j}$ is nonempty select $x_{J} \in C_{J} \cap K$. For $1 \leq j \leq n$, let $D_{j}=\overline{\operatorname{co}}\left\{x_{J}: j \in J, J \subset\{1,2, \cdots, n\}\right\}$ where $\overline{\text { co }}$ denotes the convex closure of a set. If we define $D_{n+1} \equiv C_{n+1} \equiv K$, it is clear from our construction that $C_{j} \supset D_{j}$ and that for any $J \subset\{1,2, \cdots, n+1\}, C_{J}=$ $\bigcap_{j \in J} C_{j}$ is nonempty if and only if $D_{J}$ is nonempty. It follows by Lemma 1 that there exists a retraction $R: C^{\prime}=C \cup K \rightarrow D^{\prime}=D \cup K$ such that $R(x) \in C_{j}$ if $x \in C_{j}, 1 \leq j \leq n$. Notice also that by construction $D^{\prime}=K$. Select $x_{0} \in K$ and define $H: C^{\prime} \times[0,1] \rightarrow C^{\prime}$ by

$$
\begin{aligned}
H(x, t) & =(1-2 t) x+2 t R(x), \quad 0 \leq t \leq 1 / 2, \\
& =(2-2 t) R(x)+(2 t-1) x_{0}, \quad 1 / 2 \leq t \leq 1 .
\end{aligned}
$$


Thus $C^{\prime}$ is contractible. Q.E.D.

Our next lemma is a trivial result, but we include the proof for completeness.

Lemma 3. Let $Y$ be a topological space and $f: Y \rightarrow Y$ a continuous map. Assume that there exists a subset $U \subset Y$ such that $f(U) \subset U$ and an open subset $V$ of $Y, V \subset U$, such that for eacb $y \in Y$, there exists an integer $n(y)$ such that $f^{n(y)}(y) \in V$. Then given any compact set $K \subset Y$, there exists an integer $N$ such that $f^{n}(K) \subset U$ for $n \geq N$.

Proof. For each $x \in K$ there exists an integer $n(x)$ such that $f^{n(x)}(x) \in V$. By continuity, there exists an open neighborhood $U_{x}$ of $x$ in $K$ such that $f^{n(x)}(y) \in V$ for $y \in U_{x}$. It follows that $f^{n}(y) \in U$ for $n \geq n(x)$. By the compactness of $K$, there exists a finite open covering $U_{x_{1}}, U_{x_{2}}, \cdots, U_{x_{r}}$ of $K$. For $n \geq N=$ $\max \left\{n\left(x_{1}\right), n\left(x_{2}\right), \cdots, n\left(x_{r}\right)\right\}$ and $x \in K$ we have $f^{n}(x) \in U$. Q.E.D.

Before proving our next lemma, we need to recall some algebraic generalities. Let $V$ be a vector space and $T: V \rightarrow V$ a linear endomorphism. Let $N=\{x \in V$ : $T^{n}(x)=0$ for some $\left.n \geq 1\right\}$, a linear subspace of $V$. Let $\bar{T}: V / N \rightarrow V / N$ denote the natural map induced by $T$. If $V / N$ is finite dimensional, J. Leray has defined [23] the generalized trace of $T$, which we shall write $\operatorname{tr}_{g e n}(T)$, to be the trace of $\bar{T}, \operatorname{tr}(\bar{T})$. Leray proves that this generalized trace agrees with the ordinary one when $V$ is finite dimensional. If $V$ and $W$ are vector spaces and $T: V \rightarrow W$ and $S: W \rightarrow V$ are linear endomorphisms, Leray proves that $\operatorname{tr}_{g_{\mathrm{n}}}(S T)$ is defined iff $\operatorname{tr}_{\mathrm{g} \text { en }}(T S)$ is defined and $\operatorname{tr}_{\mathrm{gen}}(S T)=\operatorname{tr}_{\mathrm{gen}}(T S)$.

A slightly different method of viewing the generatized trace (used by Browder in [6]) is sometimes convenient. Let $V$ be a vector space, let $T: V \rightarrow V$ be a linear endomorphism and let $N=\left\{x \in V: T^{n}(x)=0\right.$ for some $\left.n \geq 0\right\}$. It is not hard to prove ([26], [28]) that if $V / N$ is finite dimensional, there exists a finite dimensional subspace $E$ of $V$ such that $T(E) \subset E$ and such that for each $x \in V$ there exists an integer $m(x)$ with $T^{m(x)}(x) \in E$. Conversely, if such an $E$ exists, one can prove that $V / N$ is finite dimensional (26], [28]). Finally, one can show that $\operatorname{tr}_{\mathrm{gen}}(T)=\operatorname{tr}(T \mid E)$.

Next let $X$ be a topological space, $f: X \rightarrow X$ a continuous map and $H_{p}(X)$ the $p$ th singular homology group with coefficients in the rationals, a vector space over the rationals. As usual, $f_{*, p}: H_{p}(X) \rightarrow H_{p}(X)$, the homology map induced by $f$, is a linear endomorphism. If $\operatorname{tr}_{g \in n}\left(f_{*, p}\right)$ is defined for all $p \geq 0$ and 0 except for finitely many $p$, Leray [23] defines the generalized Lefschetz number of $f$, which we shall write $\Lambda_{g \text { e n }}(f)$, to be $\Sigma_{p \geq 0}(-1)^{p} \operatorname{tr}_{g \text { en }}\left(f_{*, p}\right)$. If $f: X \rightarrow X$ and $g: X \rightarrow X$ are homotopic in $X$ and $\Lambda_{g \text { en }}(f)$ is defined, then of course $\Lambda_{g \text { en }}(g)$ is defined and equal to $\Lambda_{g \text { en }}(f)$, since $f$ and $g$ induce the same maps in homology. If $X$ and $Y$ are topological spaces with $Y \subset X$ and if $f(X) \subset Y$, it is not hard to see that $\Lambda_{g e_{n}}(f)$ is defined iff $\Lambda_{g e n}(f \mid Y)$ is defined and $\Lambda_{g e n}(f)=\Lambda_{g e n}(f \mid Y)$, 
where $f \mid Y$ denotes $f$ viewed as a map from $Y$ to $Y$. To see this just let $g: X \rightarrow Y$ denote $f$ viewed as a map from $X$ to $Y$ and $i: Y \rightarrow X$ denote the inclusion of $Y$ into $X$. Then we have $\operatorname{tr}_{\mathrm{gen}}\left((f \mid Y)_{*, p}\right)=\operatorname{tr}_{g \mathrm{en}}\left(g_{*, p} i_{*, p}\right)=\operatorname{tr}_{\mathrm{gen}}\left(i_{*, p} g_{*, p}\right)=$ $\operatorname{tr}_{g e n}\left(f_{*, p}\right)$, hence the result. If $X$ and $Y$ are topological spaces with $Y \subset X$ and $f: X \rightarrow X$ is a continuous map such that $f(Y) \subset Y$ and $f^{n}(X) \subset Y$ for some integer $n$, then it is not hard to see by applying the above observation repeatedly that $\Lambda_{\text {gen }}(f)$ is defined iff $\Lambda_{\text {gen }}(f \mid Y)$ is and $\Lambda_{\text {gen }}(f \mid Y)=\Lambda_{g e n}(f)$ 。

With these preliminaries we can proceed to Lemma 4.

Lemma 4. Let $A$ be a topological space and $f: A \rightarrow A$ a continuous map. Let $B \subset A$ be a subspace of $A$ which is contractible in itself and assume that there exists an integer $N$ such that $f^{n}(A) \subset B$ for $n \geq N$. Then $\Lambda_{\mathrm{gen}}(f)$ is defined and equals one.

Proof. Let $V_{p}=H_{p}(A)$ and $W_{p}=\left(f_{*, p}\right)^{N}\left(V_{p}\right)$. Both $V_{p}$ and $W_{p}$ are vector spaces. Our first claim is that $W_{p}$ is zero dimensional for $p>0$ and one dimensional for $p=0$. To see this we just note that $f^{N}: A \rightarrow A$ can be written as ig, where $g$ denote $f^{N}$ viewed as a map from $A$ to $B$ and $i: B \rightarrow A$ is the inclusion. Since $B$ is contractible in itself to a point $x_{0} \in B$, this shows that $f^{N}$ (and in fact $f^{n}$ for $\left.n \geq N\right)$ is homotopic to the constant map $x \rightarrow x_{0}$. It follows that $W_{p}$ is zero dimensional for $p>0$ and one dimensional for $p=0$.

We thus see that $\Sigma_{p \geq 0}(-1) \operatorname{tr}\left(f_{*, p} \mid W_{p}\right)=\operatorname{tr}\left(f_{*, 0} \mid W_{0}\right)$. However, $f^{N+1}$ and $f^{N}$ are both homotopic to the constant map, hence homotopic, so $\left(f_{*, 0}\right)^{N+1}=$ $\left(f_{*, 0}\right)^{N}$. This shows that for any $v=\left(f_{*, 0}\right)^{N} u \in W_{0},\left(f_{*, 0}\right) v=v$, so $\operatorname{tr}\left(f_{*, 0} \mid W_{0}\right)=1$. Q.E.D.

With these lemmas we can establish our first main result. We need some further notation, however.

If $U$ is a closed subset of a Banach space $X$, let us write $U \in \mathcal{F}_{0}$ if there exists a finite number $C_{1}, C_{2}, \cdots, C_{n}$ of closed, convex subsets of $X$ such that $U=\bigcup_{i=1}^{n} C_{i}$.

Theorem 1. Let $G$ be a closed, convex subset of a Banach space $X$ and $f: G \rightarrow G$ be a continuous map. Assume that there exists a compact set $M \subset X$, a sequence of positive real numbers $\left\{r_{m}: m \geq 1\right\}$ sucb that $\lim _{m \rightarrow \infty}{ }^{r_{m}}=0$ and $a$ sequence of closed, nonempty sets $\left\{U_{m}: m \geq 1\right\}$ such that the following bold:

(1) $U_{m} \in \mathcal{F}_{0}$ and $f\left(U_{m}\right) \subset U_{m}$ for all $m$.

(2) $U_{m} \subset N_{r_{m}}(M)=\left\{x \in G: d(x, M) \leq r_{m}\right\}$.

(3) Given any compact set $K \subset G$ and any $U_{m}$, there exists an integer $N$ (depending on $K$ and $U_{m}$ ) such that $f^{N}(K) \subset U_{m}$.

Then $\Lambda_{\mathrm{gen}}\left(f \mid U_{m}\right)=1$ for all $m$, and $f$ has a fixed point.

Proof. Suppose we can prove that for each $m$ there exists $x_{m} \in U_{m}$ such that 
$\left\|f\left(x_{m}\right)-x_{m}\right\| \leq 4 r_{m}$. Since $x_{m} \in N_{r_{m}}(M), \lim _{m \rightarrow \infty} r_{m}=0$ and $M$ is compact, by taking a subsequence we can assume that $x_{m} \rightarrow x \in M \cap G$. It follows that $\|f(x)-x\|=\lim _{m \rightarrow \infty}\left\|f\left(x_{m}\right)-x_{m}\right\|=0$, so that $f(x)=x$.

Thus it suffices to find $x_{m}$ (and also prove that $\Lambda_{g e n}\left(f \mid U_{m}\right)=1$ ). Select a fixed $m$ and for notational convenience let $U_{m}=U$ and $r_{m}=r$. By assumption $U=$ $\bigcup \underset{i=1}{p} B_{i}$, where $B_{i}$ are closed, convex subsets of $G$. Let $y_{1}, y_{2}, \cdots, y_{q}$, be an $r$-net of points in $M$ (i.e., any point in $M$ is at distance less than or equal to $r$ from some $y_{j}$ ) and let $V_{2 r}\left(y_{j}\right)$ denote the closed ball of radius $2 r$ about $y_{j}$. It is easy to see that $N_{r}(M) \subset \bigcup_{j=1}^{q} V_{2 r}\left(y_{j}\right)$. Since $U \subset N_{r}(M)$, it follows that

$$
U=\left(\bigcup_{i=1}^{p} B_{i}\right) \cap\left(\bigcup_{j=1}^{q} V_{2 r}\left(y_{j}\right)\right)=\bigcup_{i, j} B_{i} \cap V_{2 r}\left(y_{j}\right) \text {. }
$$

Since diameter $\left(B_{i} \cap V_{2 r}\left(y_{j}\right)\right) \leq 4 r$ and $B_{i} \cap V_{2 r}\left(y_{j}\right)$ is closed and convex, we have shown (after reindexing and relabelling) that $U=\bigcup_{k=1}^{n} C_{k}$, where $C_{k}$ is closed and convex and diameter $\left(C_{k}\right) \leq 4 r$.

For each $J \subset\{1,2, \cdots, n\}$ such that $C_{J}=\bigcap_{k \in J} C_{k}$ is nonempty, select $x_{J} \in C_{J}$. Let $K=\overline{c o}\left\{x_{J}: J \subset\{1,2, \ldots, n\}\right\}$ and let

$$
D_{j}=\overline{\mathrm{co}}\left\{x_{J}: j \in J, J \subset\{1,2, \cdots, n\}, \quad 1 \leq j \leq n .\right.
$$

By Lemma 2, $B \equiv U \cup K$ is contractible in itself to a point. Since $K$ is compact there exists an integer $N$ such that $f^{N}(K) \subset U$; (hence $f^{n}(K) \subset U$ for $n \geq N$ ) and so if we define $A=\bigcup_{i=0}^{N-1} f^{i}(B), f: A \rightarrow A$. If we consider $f \mid A$, Lemma 4 implies that $\Lambda_{\mathrm{gen}}(f \mid A)$ is defined and equals one, since $f^{n}(A)$ lies in the contractible set $B$ for $n \geq N$. However, by our previous remarks about the generalized Lefschetz number, since $f^{N}(A) \subset U$ and $f(U) \subset U, \Lambda_{\text {ge n }}(f \mid A)=\Lambda_{\text {ge n }}(f \mid U)=1$.

If $D_{j}$ is as above for $1 \leq j \leq n$, define $D=\bigcup_{j=1}^{n} D_{j}$. It is easy to check that the hypotheses of Lemma 1 hold for $U=\bigcup_{j=1}^{n} C_{j}$ and $D=\bigcup_{j=1}^{n} D_{j}$, so there exists a retraction $R: U \rightarrow D$ such that $R\left(C_{j}\right) \subset C_{j}$ for $1 \leq j \leq n$. If we define a map $g: U \rightarrow U$ by $g(x)=(R f)(x)$, then $f$ and $g$ are homotopic in $U$ by the homotopy $t f(x)+(1-t) g(x), 0 \leq t \leq 1$. Therefore, $\Lambda_{g \text { en }}(g)$ is defined and nonzero. But $D$ is a finite union of compact, convex sets, and these are known to be compact metric ANR's. For such spaces $H_{j}(D)$ is finite dimensional and zero except for finitely many $j$, and the Lefschetz fixed point theorem holds. Therefore, we have $\Lambda_{g \text { en }}(g \mid D)=\Lambda(g \mid D) \neq 0$, where $\Lambda(g \mid D)$ is the ordinary Lefschetz number; and $g$ has a fixed point $x \in D$. Suppose that $f(x) \in C_{j}$. Then we know that $(R f)(x)=$ $x \in C_{j}$, so $\|x-f(x)\| \leq 4 r$, since diameter $\left(C_{j}\right) \leq 4 r$. Q.E.D.

Our first corollary is an easy consequence of Theorem 1 . For the case that $X$ is a Hilbert space, Corollary 1 has been proved by F. Browder (see [7, Theorem 16.3]).

Corollary 1. Let $G$ be a closed, convex subset of a Banach space $X$ and 
$f: G \rightarrow G$ a continuous map. Assume that there exists a compact set $M \subset X$ and two sequences of positive numbers $\left\{a_{k}\right\}$ and $\left\{b_{k}\right\}$ with $a_{k}>b_{k}$ and $a_{k} \rightarrow 0$ sucb that (1) for each open neighborbood $G_{0}$ of $M$ in $X$ and each $x \in G$, there exists an integer $n_{0}$ (depending on $x$ and $G_{0}$ ) such that $f^{n}(x) \in G_{0}$ for $n \geq n_{0}$ and (2) $f$ maps $N_{a_{k}}(M) \equiv\left\{x \in G: d(x, M) \leq a_{k}\right\}$ into $N_{b_{k}}(M)$ for all $k \geq 1$. Then $f$ bas a fixed point.

Proof. We take the $M$ in Theorem 1 to be the same as the $M$ above, and we define $a_{k}=r_{k}, k \geq 1$. It remains to construct the sets $U_{k}$ and verify the hypotheses of Theorem 1. Given $k \geq 1$, let $\epsilon_{k}=a_{k}-b_{k}>0$ and let $x_{i, k}, 1 \leq i \leq n(k)$, be an $\epsilon_{k}$ net for $M$. Define $C_{i, k}=\left\{x \in G:\left\|x-x_{i, k}\right\| \leq a_{k}\right\}$, a closed, convex set and define $U_{k}=\bigcup_{i=1}^{n(k)} C_{i, k}$. Clearly we have $U_{k} \subset N_{a_{k}}(M)$. On the other hand, if $y \in N_{b_{k}}(M)$, there exists $x \in M$ such that $\|y-x\| \leq b_{k}$ and there exists $x_{i, k}$ such that $\left\|x-x_{k}\right\| \leq \epsilon_{k}$, so that $\left\|y-x_{i, k}\right\| \leq a_{k}$ and $y \in U_{k}$. It follows that $N_{b_{k}}(M) \subset$ $U_{k}$, and this implies that $f\left(U_{k}\right) \subset U_{k}$ and $U_{k}$ contains an open neighborhood of $M$. By Lemma 3, given any compact set $K \subset G$, there exists an integer $N$ such that $f^{N}(K) \subset U_{k}$. Thus hypothesis (3) of Theorem 1 is satisfied, and we have already verified hypotheses (1) and (2). Q.E.D.

Corollary 2 (see [13]). Let $G$ be a closed, convex subset of a Banach space $X$ and $f: G \rightarrow G$ a continuous map. Assume there exists a compact, nonempty set $M \subset X$ and a constant $c<1$ such that for all $x \in G, d(f(x), M) \leq c d(x, M)$. Then $f$ has a fixed point.

Proof. In the notation of Corollary 1 , let $a_{k}=1 / k$ and $b_{k}=c a_{k}$ for integers $k \geq 1$. Then it is clear the hypotheses of Corollary 1 hold. Q.E.D.

Before stating our next corollary we need to introduce some definitions.

Definitions. Let $Y$ be a topological space, $f: Y \rightarrow Y$ a map, and $M$ a subset of $Y$. We say that " $M$ is an attractor for compact sets under $f$ " if (1) $M$ is compact, nonempty and $f(M) \subset M$, and (2) given any compact set $A \subset Y$ and any open neighborhood $U$ of $M$, there exists an integer $N$ (depending on $A$ and $U$ ) such that $f^{n}(A) \subset U$ for $n \geq N$. We say that " $M$ is an attractor for points under $f$ " if (1) above holds and given any point $y \in Y$ and any open neighborhood $U$ of $M$, there exists an integer $N$ (depending on $y$ and $U$ ) such that $f^{n}(y) \in U$ for $n \geq N$.

One encounters attractors in analysis when one considers the map of translation along trajectories of differential equations or functional differential equations which satisfy various assumptions of stability. For our immediate purposes, however, the reason for introducing this notion is the following simple lemma:

Lemma 5. Let $Y$ be a metric space and $f: Y \rightarrow Y$ a cont inuous map. Assume that there exists a set $M \subset Y$ which is an attractor for compact sets under $f$. Then given any open neighborbood $U$ of $M$, there exists an open neighborbood $V$ of $M$ such that $V \subset U$ and $f(V) \subset V$. 
Proof. Let $V=\left\{x \in U: f^{n}(x) \in U\right.$ for all $\left.n \geq 1\right\}$. Clearly, $M \subset V \subset U$ and $f(V) \subset$ $V$. It remains to show $V$ is open. Suppose not. Then there exists a point $x \in V$ and a sequence $x_{k} \rightarrow x$ such that $x_{k} \notin V$ for all $k$. Since $x_{k} \notin V$, there exists a first integer $n(k)$ such that $f^{n(k)}\left(x_{k}\right) \notin U$. Since $f^{n}(x) \in V$ for all $n \geq 0$ and since $x_{k} \rightarrow x$, it follows by the continuity of $f$ that $\lim _{k \rightarrow \infty} n(k)=\infty$. On the other hand, if $A=\left\{x_{k}: k \geq 1\right\} \cup\{x\}, A$ is compact, so $f^{n}(A) \subset U$ for all $n \geq N=$ an integer depending on $A$ and $U$. This contradicts the choice of $A$. Q.E.D.

Before stating our next lemma we need to recall more notation and definitions.

Definitions. Let $Y$ be a topological space and $f: Y \rightarrow Y$ a map. If $G$ is a subset of $Y$, define $C_{1}(f, G)=f(G), C_{n}(f, G)=f\left(G \cap C_{n-1}(f, G)\right)$ for $n \geq 2$ and $C_{\infty}(f, G)=\bigcap_{n \geq 1} C_{n}(f, G) ; C_{\infty}(f, Y)=\bigcap_{n \geq 1} f^{n}(Y)$ is called the "core of $f . "$ If $A$ is a subset of $Y, \cup_{n \geq 0} f^{n}(A) \equiv O(A)$ is called the "orbit of $A$ under $f . "$

The idea of looking at the core of a map in the context of fixed point theory goes back at least as far as the work of J. Leray on the fixed point index ([21], [22]). Lemma 6 below appears to be due A. Gleason and R. S. Palais (unpublished); F. Browder also establishes this result in [6]. We give a proof only for completeness.

Lemma 6 (Gleason and Palais). Let $Y$ be a metric space and $f: Y \rightarrow Y$ a continuous map. Assume that $C_{\infty}(f, Y)$, the core of $f$, bas compact closure in $Y$ and that the orbit of any point $y \in Y$ bas compact closure. Finally assume that there exists an open neighborbood $V$ of $M \equiv \mathrm{cl}\left(C_{\infty}(f, Y)\right)$ such that $\mathrm{cl}(f(V))$ is compact. Then $M$ is an attractor for compact sets under $f$, and the orbit of any compact set under $f$ has compact closure.

Proof. First let us show that $M$ is an attractor for points. Thus let $U$ be any open neighborhood of $M$ and $y$ be a point in $Y$ and assume that there does not exist an integer $N$ such that $f^{n}(y) \in U$ for $n \geq N$. By assumption $A=\mathrm{cl}(O(y))$ is compact and obviously $f(A) \subset A$. It follows that $\left\{f^{j}(A) \cap(Y-U)\right\}$ is a decreasing sequence of nonempty compact sets, so that $\left(\bigcap_{j \geq 1} f^{j}(A)\right) \cap(Y-U)$ is nonempty ( $Y-U$ denotes the complement of $U$ in $Y$ ). However, by the definition of $M$, we must have $\bigcap_{j \geq 1} f^{j}(A) \subset M \subset U$, a contradiction, so that $M$ must be an attractor for points.

Let $V$ be as in the statement of the lemma. For each $x \in \mathrm{cl}(f(V))$ there exists a positive integer $m(x)$ and an open neighborhood $U(x)$ of $x$ in $\mathrm{cl}(f(V))$ such that $f^{m(x)}(y) \in V$ for $y \in U(x)$. Since $\operatorname{cl}(f(V))$ is compact, we can write $\operatorname{cl}(f(V))=$ $\bigcup_{i=1}^{r} U\left(x_{i}\right)$. We define $m=\max _{1 \leq i \leq r}\left\{m\left(x_{i}\right)\right\}$ and $\tilde{V}=\bigcup_{j=0}^{m} f^{j}(V)$, and we claim that $f(\tilde{V}) \subset \tilde{V}$. To show $f(\tilde{V}) \subset \widetilde{V}$ it suffices to show that if $y=f^{m-1}(x)$ for $x \in f(V)$, then $f(y) \in \tilde{V}$. But $x \in U\left(x_{i}\right)$ for some $i$ so that

$$
f(y)=f^{m-m\left(x_{i}\right)}\left(f^{m\left(x_{i}\right)}(x)\right) \in f^{m-m\left(x_{i}\right)}(V) \subset \tilde{V} .
$$


Assume now that $B \subset Y$ is a compact set. We wish to show that $O(B)$ has compact closure and to do that it suffices to show that $O(B)$ is contained in a compact set. Since $M$ is an attractor for points, for each $x \in B$ there exists an integer $n(x)$ such that $f^{j}(x) \in V$ for $j \geq n(x)$. By continuity there exists an open neighborhood $V(x)$ such that $f^{n(x)}(y) \in \bar{V}$ for $y \in V(x)$. Consequently we have $f^{j}(y) \in \tilde{V}$ that for $y \in V(x)$ and $j \geq n(x)$. By the compactness of $B, B \subset \bigcup_{i=1}^{r} V\left(x_{i}\right)$, so that if $n=\max \left\{n\left(x_{i}\right)\right\}, f^{j}(B) \subset \widetilde{V}$ for $j \geq n$. We thus see that

$$
\operatorname{cl}(O(B)) \subset \bigcup_{j=0}^{n} f^{j}(B) \cup \mathrm{cl}(f(\tilde{V})),
$$

a compact set.

It remains to show that $M$ is an attractor for compact sets. To see this, let $B$ be a compact set and let $A_{1}=\mathrm{cl}(O(B))$. Then if we assume $M$ is not an attractor of compact sets (in particular, say not an attractor of $B$ ), the same argument given in the first paragraph of the proof leads to a contradiction. Q.E.D.

Actually the hypotheses of Lemma 6 are unnecessarily restrictive. One can easily check that the proof of Lemma 6 implies the following slightly more general result.

Lemma 7. Let $Y$ be a metric space and $f: Y \rightarrow Y$ a continuous map. Assume that the orbit of any $y \in Y$ has compact closure. Suppose that there exists a compact set $M \subset Y$ sucb that $f(M) \subset M$ and such that $C_{\infty}(f, K) \subset M$ for any compact set $K \subset Y$ for which $f(K) \subset K$. Finally assume that there exists an open neighborbood $V$ of $M$ such that $\mathrm{cl}(f(V))$ is compact. Then $M$ is an attractor for compact sets and the orbit of any compact set has compact closure.

Our next lemma is also a result of Gleason and Palais (unpublished). A proof of a slightly less general version is given by Browder in [6]. Again, we give a proof only for the sake of completeness.

Lemma 8 (Gleason and Palais). Let bypotheses and notation be as in Lemma 7. Then given any open neighborbood $W$ of $M$, there exists an open neigbborbood $U$ of $M$ such that $U \subset W$ and $\mathrm{cl}(f(U)) \subset U$.

Proof. Let $W^{\prime}$ be an open neighborhood of $M$ such that $\mathrm{cl}\left(W^{\prime}\right) \subset W \cap V$. By Lemma 5 and Lemma 7 there exists an open neighborhood $U_{0}$ of $M$ such that $U_{0} \subset W^{\prime}$ and $f\left(U_{0}\right) \subset U_{0}$. Let $A=\operatorname{cl}\left(f\left(U_{0}\right)\right)$, a compact subset of $\operatorname{cl}\left(U_{0}\right)$. Since (by Lemma 7) $M$ is an attractor of compact sets, there exists an integer $N$ such that $f^{N}(A) \subset U_{0}$. (Notice that $f^{j}(A) \subset \mathrm{cl}\left(U_{0}\right)$ for all $j \geq 0$, since $f\left(\operatorname{cl}\left(U_{0}\right)\right) \subset$ $\left.\operatorname{cl}\left(U_{0}\right)_{.}\right)$Let $U_{N}$ be an open neighborhood of $f^{N-1}(A)$ such that $U_{N} \subset V \cap W$ and such that $\operatorname{cl}\left(f\left(U_{N}\right)\right) \subset U_{0}$ (since $f^{N}(A)$ is a compact subset of $U_{0}$ ). Generally, if $U_{j}$ is an open neighborhood of $f^{j-1}(A)$ for $1<j \leq N-1$, let $U_{j-1}$ be an open neighborhood of $f^{j-2}(A)$ such that $U_{j-1} \subset V \cap W$ and $\mathrm{cl}\left(f\left(U_{j-1}\right)\right) \subset U_{j}$. In this 
way we obtain open neighborhoods $U_{j}$ of $f^{j-1}(A)$ for $1 \leq j \leq N$ such that $U_{j} \subset V \cap W$ and $\operatorname{cl}\left(f\left(U_{j}\right)\right) \subset U_{j+1}$ (with the convention that $U_{0}=U_{N+1}$ ). Defining $U=$ $\bigcup_{j=0}^{N} U_{j}$, it is not hard to show that $U$ satisfies the conditions of Lemma 8. Q.E.D.

We are now in a position to obtain our next corollary of Theorem 1 . This result was essentially established by F. E. Browder in [6] and has been proved by Browder in the full generality below in [7]. A somewhat less general theorem is given by $\mathrm{H}$. Steinle in in his dissertation [32].

Corollary 3 (Browder [6], [7]). Let $G$ be a closed, convex subset of a Banach space $X$ and $f: G \rightarrow G$ a continuous map. Assume that (1) $\bigcap_{n \geq 1} f^{n}(G)$, the core of $f$, has compact closure in $G$. (2) For each $x \in G$, the orbit of $x$ under $f$ has compact closure. (3) There exists an open neighborbood $V$ of $\operatorname{cl}\left(\bigcap_{n \geq 1} f^{n}(G)\right)$ such that $\mathrm{cl}(f(V))$ is compact. Then $f$ bas a fixed point.

Proof. By Lemma 8 there exists an open neighborhood $U$ of $A=$ $\operatorname{cl}\left(\bigcap_{n \geq 1} f^{n}(G)\right)$ such that $U \subset V$ and $\operatorname{cl}(f(U)) \subset U$. Let $M=A \cup \mathrm{cl}(f(U))$, a compact subset of $U$. By Lemma $8, M$ is an attractor for compact sets under $f$. Since $M$ is compact, there exists a real number $a>0$ such that $N_{a}(M) \subset U$. Let $\left\{a_{k}\right\}$ be any sequence of positive numbers such that $a_{k} \leq a$ for all $k$ and $\lim a_{k}=0$. If $\left\{b_{k}\right\}$ is any sequence of positive numbers such that $b_{k}<a_{k}$, we have $f\left(N_{a_{k}}(M)\right) \subset$ $N_{b_{k}}(M)$; in fact we have $f\left(N_{a}(M)\right) \subset M$. Thus the hypotheses of Corollary 1 are satisfied and $f$ has a fixed point. Q.E.D.

An examination of the proof of Corollary 3 shows that the same proof gives the following somewhat more general result:

Corollary 4. Let $G$ be a closed, convex sutset of a Banach space $X$ and f: $G \rightarrow G$ a continuous map. Assume that (1) There exists a compact set $A \subset G$ such that $f(A) \subset A$ and such that $C_{\infty}(f, K) \subset A$ for any compact set $K \subset G$ for which $f(K) \subset K$. (2) For each $x \in G$, the orbit of $x$ under $f$ has compact closure. (3) There exists an open neighborbood $V$ of $A$ sucb that $\mathrm{cl}(f(V))$ is compact. Then $f$ has a fixed point.

One can obtain one of W. A. Horn's results in [16] as a consequence of Corollary 4.

Corollary 5 (W. A. Horn [16]). Let $G$ be a closed, convex subset of a Banach space $X$ and $f: G \rightarrow G$ a compact map ( $f$ is continuous and takes bounded sets into precompact sets). Assume there exists a bounded set $E$ such that for each $x \in G$ there exists an integer $m(x)=m$ such that $f^{m}(x) \in E$. Then $f$ bas a fixed point.

Proof. Let $V$ be a bounded open neighborhood of $\mathrm{cl}(E)$ and let $K=\mathrm{cl}(f(V))$, 
a compact set. For each $x \in K$, there exists a positive integer $m(x)$ and an open neighborhood $U(x)$ of $x$ in $K$ such that $f^{m(x)}(y) \in V$ for $y \in U(x)$. Let $\left\{U\left(x_{i}\right): 1 \leq\right.$ $i \leq r\}$ be an open covering of $K$ and let $m=\max \left\{m\left(x_{i}\right)\right\}$. Just as in the proof of Lemma 6 we see that if $\tilde{V}=\bigcup_{j=0}^{m} f^{j}(V), f(\tilde{V}) \subset \tilde{V}$.

Define $A=\operatorname{cl}(f(\tilde{V}))$, so that $A$ is a compact set and $f(A) \subset A$. We wish to show that with this $A$ the hypotheses of Corollary 4 hold. It suffices to show that if $K$ is any compact set in $G$, there exists an integer $N$ (depending on $K$ ) such that $f^{n}(K) \subset A$ for $n \geq N$. For each $x \in K$, there exists an integer $n(x)$ and an open neighborhood $O(x)$ such that $f^{n(x)}(y) \in V$ for $y \in O(x)$. It follows that $f^{j}(y) \in A$ for $j \geq n(x)+1$. Since $K$ is compact there exists a finite open covering $\left\{O\left(x_{i}\right): 1 \leq\right.$ $i \leq s\}$ of $K$, and if $N=\max \left\{n\left(x_{i}\right)+1\right\}, f^{n}(K) \subset A$ for $n \geq N$. Q.E.D.

2. In this section we wish to obtain some less straightforward consequences of Theorem 1. We begin by recalling the notion of measure of noncompactness of a bounded metric space. This is a very useful idea which was first introduced by C. Kuratowski [19].

Definition. Let $(Y, \rho)$ be a bounded metric space. The measure of noncompactness of $Y, \gamma(Y)$, equals $\inf \left\{d>0\right.$ : there exists a finite number of sets $S_{1}$, $S_{2}, \cdots, S_{n}$ such that $Y=\bigcup_{i=1}^{n} S_{i}$ and diameter $\left.\left(S_{i}\right) \leq d\right\}$.

Of course if $Y$ is a bounded complete metric space, $\gamma(Y)=0$ if and only if $Y$ is compact-hence the name measure of noncompactness. Kuratowski establishes a number of properties of the measure of noncompactness; of these results the following proposition will prove most useful for our purposes:

Proposition 1 (Kuratowski [19]). Let $(Y, \rho)$ be a complete metric space and let $Y_{1} \supset Y_{2} \supset \ldots \supset Y_{n} \supset \ldots$ be a decreasing sequence of closed, bounded, nonempty subsets of $Y$ (which inberit their metrics from $Y$ ). Assume that $\lim _{n \rightarrow \infty} \gamma\left(Y_{n}\right)=0$. Then $Y_{\infty} \equiv \bigcap_{n \geq 1} Y_{n}$ is a nonempty compact set, and if $U$ is any open neighborbood of $Y_{\infty}$, there exists an integer $N$ (depending on $U$ ) such that $Y_{n} \subset U$ for $n \geq N$.

If $Y_{1}$ and $Y_{2}$ are metric spaces and $f: Y_{1} \rightarrow Y_{2}$ is a continuous map, Kuratowski also introduces a class of maps which we shall call " $k$-set-contractions." Specifically, $f$ is called a $k$-set-contraction if for every bounded subset $A$ of $Y_{1}$, $f(A)$ is bounded and $\gamma_{2}(f(A)) \leq k \gamma_{1}(A)$. In the work below $Y_{1}$ will always be a subset of a Banach space $Y_{2}$, from which $Y_{1}$ inherits its metric. If $U$ is a subset of a Banach space $X, g: U \rightarrow X$ is a Lipschitz map with constant $k$, and $C: U \rightarrow$ $X$ is a compact map, then $f=g+C$ is a $k$-set-contraction. This is perhaps the simplest nontrivial example of a $k$-set-contraction. More general examples are given in [26] and [28]. For instance, it is shown in [26] that the radial retraction onto a closed ball in an infinite dimensional Banach space $X$ is a 1-set-contraction, even though DeFigureido and Karlovitz have shown (Bull. Amer. Math. Soc. 
73 (1967), 364-368) that it is a Lipschitz map with constant one if and only if $X$ is a Hilbert space. Also one can take compositions and use partition of unity arguments with $k$-set-contractions.

If $X$ is a Banach space $G$. Darbo has shown that the measure of noncompactness satisfies other properties related to the linear structure of $X$. Specifically, if $A$ and $B$ are subsets of $X$, define $A+B=\{a+b: a \in A, b \in B\}, \operatorname{co}(A)=$ the convex hull of $A$ (the smallest convex set containing $A$ ) and $\overline{c o}(A)=$ the closed convex hull of $A$.

Proposition 2 (Darbo [8]). Let $A$ and $B$ be bounded subsets of a Banach space $X$. Then $\gamma(A+B) \leq \gamma(A)+\gamma(B)$ and $\gamma(\overline{\mathrm{co}} A)=\gamma(A)$.

Using Proposition 2, Darbo establishes the following fixed point theorem, which is the starting point for the results of this section.

Proposition 3 (Darbo [8]). Let $G$ be a closed, bounded convex set and let $f$ : $G \rightarrow G$ be a k-set-contraction, $k<1$. Then $f$ has a fixed point.

The goal here is to generalize Proposition 3, but further mathematical apparatus is needed. We need to recall the notion of the fixed point index and some of its basic properties. Thus let $A$ be a compact metric ANR. If $A$ is a compact subset of a Banach space and $A=\bigcup_{i=1}^{n} C_{i}$, where the $C_{i}$ are compact, convex subsets of $X, A$ is a compact metric ANR. This is the most important example for our purposes. Let $G$ be an open subset of $A$ and let $f: G \rightarrow A$ be a continuous map such that $S=\{x \in G: f(x)=x\}$ is compact (possibly empty). Then there is an integer defined, $i_{A}(f, G)$, called the fixed point index of $f$ on $G$. Roughly speaking, $i_{A}(f, G)$ is the number of fixed points of $f$ in $G$ counted algebraically. If $i_{A}(f, G) \neq 0$, then $f$ has a fixed point in $G$; and ir $U$ is any open neighborhood of $S, U \subset G$, then $i_{A}(f, U)=i_{A}(f, G)$. If $B$ is a compact metric ANR contained in $A$ and if $f(G) \subset B$, then the fixed point index respects this relation and $i_{A}(f, G)=$ $i_{B}(f, G \cap B)$. Finally, the fixed point index agrees with the Lefschetz number when both are defined, i.e., if $G=A, i_{A}(f, A)=\Lambda(f)$, the Lefschetz number of $f$. The fixed point index satisfies other properties, e.g. the homotopy property, and in fact the index can be axiomatically defined by four properties; but the results given here will suffice for our purposes. We refer the reader to [3], [10], [21], [30] or [33] for more details.

The fixed point index described briefly above can be defined for $k$-set-contractions, $k<1$, defined on open subsets of "nice" metric (noncompact) ANR'sand in fact for more general maps. All the properties of the classical fixed point index have direct generalizations in this context; and in fact one can give four properties which again determine this generalized fixed point index axiomatically. We refer the reader to [24] for a summary and to [26] or [28] for details. 
All we shall need below is the definition of the generalized fixed point index, though we should remark that its usefulness stems from the properties it satisfies. To begin the definition, if $V$ is a subset of a Banach space $B$ and $f: V \rightarrow B$ is a map, define $K_{1} \equiv K_{1}(f, V)=\overline{c o} f(V)$, where $\overline{c o}$ denotes convex closure. Generally, for $n>1$, define $K_{n} \equiv K_{n}(f, V) \equiv \overline{c o} f\left(V \cap K_{n-1}\right)$ and define $K_{\infty}=K_{\infty}(f, V)=\bigcap_{n \geq 1} K_{n}$. It is easy to see that $f\left(V \cap K_{\infty}\right) \subset K_{\infty}$. Now suppose that $V$ is bounded and $f$ is a $k$-set-contraction, $k<1$. By using previously mentioned properties, one can prove that $\gamma\left(K_{n}\right) \rightarrow 0$ (in fact $\gamma\left(K_{n}\right) \leq k^{n} \gamma(V)$ ), so that by Proposition $1, K_{\infty}$ is compact and $K_{\infty}$ is empty if and only if $K_{n}$ is empty for some $n$.

Next suppose that $A$ is a closed subset of a Banach space $X$. We shall write $A \in \mathcal{F}$ and say that $A$ has a locally finite cover by closed, convex sets if there exists a family of closed, convex subsets of $X,\left\{C_{i}: i \in I\right\}$, such that $A=\bigcup_{i \in I} C_{i}$ and given any $x \in A$ there exists an open neighborhood $U_{x}$ of $x$ such that $U_{x} \cap C_{i}$ is empty except for finitely many $i$. One can show that if $A \in \mathcal{F}, A$ is a metric ANR.

With the se definitions, assume that $A \in \mathcal{F}, V$ is a bounded open subset of $A$ and $f: V \rightarrow A$ is a $k$-set-contraction with $k<1$. Assume that $S=\{x \in V: f(x)=$ $x\}$ is compact (possibly empty) and write $K_{\infty}^{*}=K_{\infty}(f, V) \cap A$, so that $K_{\infty}^{*}$ is a finite union of compact, convex sets. We define $i_{A}(f, V)$, the generalized fixed point index of $f$ on $V$, to be $i_{K_{\infty}^{*}}^{*}\left(f, V \cap K_{\infty}^{*}\right)$. One can prove that this definition is consistent with the classical fixed point index: if $A$ above is compact, so that the classical fixed point index is already defined, $i_{A}(f, V)=i_{K_{\infty}}^{*}\left(f, V \cap K_{\infty}^{*}\right)$. Just as for the ordinary fixed point index, if $i_{A}(f, V) \neq 0, f$ has a fixed point, and if $W$ is any open neighborhood (in $A$ ) of $S, W \subset V$, then $i_{A}(f, V)=i_{A}(f, W)$.

Now we can begin work on further consequences of Theorem 1 . Our next lemma is another point set topology result.

Lemma 9. Let $(Y, d)$ be a complete metric space and $f: Y \rightarrow Y$ a continuous map. Assume that there exists a compact set $M \subset Y$ which is an attractor for compact sets under $f$. Suppose also that there exists a bounded, open neighborbood $V$ of $M$, such that $\lim _{j \rightarrow \infty} \gamma\left(f^{j}(V)\right)=0$. Then given any open neighborbood $W$ of $M$, there exists an open neighborbood $U$ of $M, U \subset W$, sucb that $\mathrm{cl}(f(U)) \subset U$. Furthermore, if $U$ is any open neighborbood of $M$ such that $U \subset V$ and $f(U) \subset U$ and $O$ is an open neighborbood of $M$, there exists an integer $N$ (depending on $O$ and $U$ ) sucb that $\operatorname{cl}\left(f^{n}(U)\right) \subset O$ for $n \geq N$.

Proof. Begin with the second assertion, so suppose that $U$ is an open neighborhood of $M$ contained in $V$ and $f(U) \subset U$. If we let $A_{n}=\operatorname{cl}\left(f^{n}(U)\right)$, then by the assumptions $A_{n}$ is a decreasing sequence of closed, nonempty sets such that $\gamma\left(A_{n}\right) \rightarrow 0$, so by Proposition $1, A_{\infty}=\bigcap_{n \geq 1} A_{n}$ is nonempty and compact. We claim that $f\left(A_{\infty}\right)=\left\{f(y): y \in A_{\infty}\right\}=A_{\infty}$. For suppose not. Then there exists $\epsilon>0$ and $y \in A_{\infty}$ such that $y \notin N_{\epsilon}\left(f\left(A_{\infty}\right)\right)=\left\{x \in Y: d\left(x, f\left(A_{\infty}\right)\right) \leq \epsilon\right\}$. By continuity and 
compactness, there exists $\delta>0$ such that $f\left(N_{\delta}\left(A_{\infty}\right)\right) \subset N_{\epsilon}\left(f\left(A_{\infty}\right)\right)$. By Proposition 1 again, $A_{n} \subset N_{\delta}\left(A_{\infty}\right)$ for $n \geq n_{0}$, so that $A_{n+1} \subset \operatorname{cl}\left(f\left(A_{n}\right)\right) \subset N_{\epsilon}\left(f\left(A_{\infty}\right)\right)$ for $n \geq n_{0}$. But this implies $A_{\infty} \subset N_{\epsilon}\left(f\left(A_{\infty}\right)\right)$ a contradiction. It follows that $f\left(A_{\infty}\right)=A_{\infty}$, so $f^{N}\left(A_{\infty}\right)=A_{\infty}$ for all positive integers $N$. But $M$ is an attractor so for some integer $N, f^{N}\left(A_{\infty}\right)=A_{\infty} \subset O$. Since $A_{\infty}$ is compact, there exists a positive number $r$ such that $N_{r}\left(A_{\infty}\right) \subset O$. By Proposition $1 A_{n} \subset N_{r}\left(A_{\infty}\right)$ for $n \geq n_{1}$, which proves the second assertion.

Now let $W$ be any open neighborhood of $M$. By Lemma 5 , there exists an open neighborhood $U_{0}$ of $M$ such that $f\left(U_{0}\right) \subset U_{0}$ and such that $\operatorname{cl}\left(U_{0}\right) \subset V \cap W$. By the above remarks there exists an integer $N$ such that $\operatorname{cl}\left(f^{n}\left(U_{0}\right)\right) \subset U_{0}$ for $n \geq N$. Let $U_{N-1}$ be an open neighborhood of $\mathrm{cl}\left(f^{N-1}\left(U_{0}\right)\right)$ in $V \cap W$ such that $\operatorname{cl}\left(f\left(U_{N-1}\right)\right) \subset U_{0}$ and proceed inductively, defining $U_{N}=U_{0}$ : If sets $U_{j}$ have been constructed for $r \leq j \leq N-1$ such that $U_{j}$ is an open neighborhood of $\operatorname{cl}\left(f^{j}\left(U_{0}\right)\right)$ in $V \cap W$ and $\operatorname{cl}\left(f\left(U_{j}\right)\right) \subset U_{j+1}$ for $r \leq j \leq N-1$, define $U_{r-1}$ to be an open neighborhood of $\operatorname{cl}\left(f^{r-1}\left(U_{0}\right)\right)$ in $V \cap W$ such that $\operatorname{cl}\left(f\left(U_{r-1}\right)\right) \subset U_{r}$. In this way we obtain an open neighborhood $U=\bigcup_{j=0}^{N-1} U_{j}$ of $M$ such that $\operatorname{cl}(f(U)) \subset U$ and $U \subset V \subset W$. Q.E.D.

With the aid of Lemma 9 we can obtain our next corollary of Theorem 1. Corollary 6 is closely related to the results in [6], [25], but it does not seem directly comparable. The proof given below is in two parts: the first part shows the existence of a fixed point, and the second part shows that an appropriate fixed point index equals one.

Corollary 6. Let $G$ be a closed, convex subset of a Banach space $X$ and $f$ : $G \rightarrow G$ a continuous map. Assume that there exists a compact set $M \subset G$ which is an attractor for compact sets under $f$ and suppose that there is an open neighborbood $V$ of $M$ such that $f \mid V$ is a k-set-contraction, $k<1$. Then $f$ bas a fixed point and $i_{G}(f, V)=1$.

Proof. By Lemma 5 there exists a bounded open neighborhood $W$ of $M, W \subset V$, such that $f(W) \subset W$. Since $\gamma\left(f^{j}(W)\right) \leq k^{j} \gamma(W)$, the conditions of Lemma 9 apply and there exists an open neighborhood $U$ of $M, U \subset W$, such that $\operatorname{cl}\left((f(U)) \subset U\right.$. Let $K_{n}=$ $K_{n}(f, U)$ for $n \geq 1$ and $K_{\infty}=K_{\infty}(f, U)$ and notice that since $\gamma\left(K_{n}\right) \leq k^{n} \gamma(U), K_{\infty}$ is compact and $K_{n}$ approaches $K_{\infty}$ in the Hausdorff metric. Because $\mathrm{cl}\left(f\left(K_{\infty} \cap U\right)\right)$ is compact and contained in $U$, there exists $\delta>0$ such that $N_{3 \delta}\left(f\left(K_{\infty} \cap U\right)\right) \subset U$. Take $\epsilon>0$ such that $f\left(N_{\epsilon}\left(K_{\infty} \cap U\right)\right) \subset N_{\delta}\left(f\left(K_{\infty} \cap U\right)\right)\left(N_{\delta}\right.$ indicates the closed $\delta$ neighborhood) and by Proposition 1 let $n_{0}$ be an integér such that $K_{n} \cap U \subset$ $N_{\epsilon}\left(K_{\infty} \cap U\right)$ for $n \geq n_{0}$. It then follows that $N_{2 \delta}\left(f\left(U \cap K_{n}\right)\right) \cap K_{n} \subset U \cap K_{n}$. Since $\gamma\left(K_{n}\right) \rightarrow 0$, there exists an integer $n_{1}$ such that $\gamma\left(f\left(U \cap K_{n}\right)\right)<2 \delta$ for $n \geq n_{1}$. For $n \geq \max \left\{n_{0}, n_{1}\right\}=n_{2}$, let $f\left(U \cap K_{n}\right)=\bigcup_{j=1}^{m(n)} S_{j, n}$, where diameter $\left(S_{j, n}\right)<2 \delta$. If we define $U_{j, n}=\overline{\mathrm{co}}\left(S_{j, n}\right)$ and $U_{n}=\bigcup_{j=1}^{m(n)} U_{j, n}$, it is clear from our construction 
that $f\left(U \cap K_{n}\right) \subset U_{n} \subset U \cap K_{n}$, so that $f\left(U_{n}\right) \subset U_{n}$ and of course $U_{n} \in \mathcal{F}_{0}$. If we define $M_{1}=M \cup \mathrm{cl}\left(K_{\infty} \cup U\right), M_{1}$ is attractor for compact sets under $f$ and it is easy to check that the hypotheses of Theorem 1 hold with $M_{1}$ and $\left\{U_{n}\right\}$. Therefore $f$ has a fixed point.

It remains to show that $i_{G}(f, V)=1$. By Theorem 1 we know that $\Lambda_{\text {gen }}\left(f \mid U_{n}\right)=$ 1. By construction we have $f^{n+1}(U) \subset U_{n}$, so the properties of the generalized Lefschetz number imply that $\Lambda_{g \text { en }}(f \mid U)=\Lambda_{g \text { e } n}\left(f \mid U_{n}\right)=1$. Just as we showed above, there exist compact, convex sets $T_{1}, T_{2}, \cdots, T_{m}$ such that $\mathrm{cl}\left(f\left(U \cap K_{\infty}\right)\right)$ $\subset \bigcup_{i=1}^{m} T_{i} \subset U \cap K_{\infty}$. It is not hard to see that there exists $r>0$ such that if $N_{r}\left(T_{i}\right)=\left\{x \in G: d\left(x, T_{i}\right) \subset r\right\}, N_{r}\left(T_{i}\right) \subset U$ for $1 \leq i \leq m$ and $\bigcap_{j \in J} N_{r}\left(T_{j}\right)=\varnothing$ if and only if $\bigcap_{j \in J} T_{j}=\varnothing$ for every subset $J \subset\{1,2, \cdots, m\}$. Since $\bigcup_{i=1}^{m^{j}} N_{r}\left(T_{i}\right)$ contains an open neighborhood of $A_{\infty}=\bigcup_{i=1}^{m} T_{i}$, the usual argument shows that there exists an integer $n_{0}$ such that

$$
f\left(U \cap K_{n}\right) \subset \bigcup_{i=1}^{m} N_{r}\left(T_{i}\right) \text { for } n \geq n_{0} .
$$

If we define $A_{i, n}=N_{r}\left(T_{i}\right) \cap K_{n}$ and $A_{n}=\bigcup_{i=1}^{m} A_{i, n}$, it is not hard to see that, for $n \geq n_{0}, f\left(U \cap K_{n}\right) \subset A_{n} \subset U \cap K_{n}$, so that $f\left(A_{n}\right) \subset A_{n}$. Also, since by our construction $\bigcap_{j \in J} \dot{A_{j, n}}=\varnothing$ if and only if $\bigcap_{j \in J} T_{j}=\varnothing$ for every $J \subset\{1,2, \cdots, m\}$, by Lemma 1 there exists a retraction $R_{n}: A_{n} \rightarrow A_{\infty}$ such that $R_{n}\left(A_{i, n}\right) \subset A_{i, n}$ for $1 \leq i \leq m$.

We are now almost done. Since $f^{n+1}(U) \subset A_{n}, \Lambda_{g e n}(f \mid U)=\Lambda_{\text {ge } n}\left(f \mid A_{n}\right)=1$. But because $R_{n} f$ and $f$ are homotopic in $A_{n}$ and $\left(R_{n} f\right)\left(A_{n}\right) \subset A_{\infty}$,

$$
\Lambda_{\text {gen }}\left(f \mid A_{n}\right)=\Lambda_{\text {gen }}\left(R_{n} f \mid A_{n}\right)=\Lambda_{\text {gen }}\left(R_{n} f \mid A_{\infty}\right)=\Lambda\left(f \mid A_{\infty}\right) .
$$

However, we know that $f\left(U \cap K_{\infty}\right) \subset A_{\infty}$, so by one of the basic properties of the fixed point index, $\Lambda\left(f \mid A_{\infty}\right)=i_{K_{\infty}}\left(f, U \cap K_{\infty}\right) \equiv i_{G}(f, U)$. Thus we see that $i_{G}(f, U)=\Lambda_{\mathrm{gen}}(f \mid U)=1$, so that by one of the properties of the generalized fixed point index, $i_{G}(f, V)=i_{G}(f, U)=1$. Q.E.D.

The same proof used above actually establishes a slightly more general result. Colollary $6^{\prime}$ below is of interest in that its hypotheses are independent of equivalent norms on the overlying Banach space $X$, and this is not true of Corollary 6 itself.

Corollary 6'. Let $G$ be a closed, convex subset of a Banach space $X$ and f: $G \rightarrow G$ a continuous map. Assume that there exists a compact set $M$ which is an attractor for compact sets under $f$ and an open neighborbood $V$ of $M$ such that $\lim _{n \rightarrow \infty} \gamma\left(K_{n}(f, V)\right)=0$. Then if $K_{\infty}=K_{\infty}(f, V), i_{K_{\infty}}\left(f, V \cap K_{\infty}\right)=1$ and $f$ has a fixed point.

A number of results follow in a straightforward way from Corollary 6 . The 
following is a typical consequence.

Corollary 7. Let $G$ be a closed, convex subset of a Banach space $X$ and f: $G \rightarrow G$ a k-set-contraction, $k<1$. Assume that there exists a bounded set $E \subset G$ such that $f(E) \subset E$ and such that given any compact set $A \subset G$, there exists an integer $N$ (depending on $A$ ) such that $f^{N}(A) \subset E$. Then $f$ has, a fixed point, and if $V$ is any bounded open neighborbood of the (compact) fixed point set of $f$, $i_{G}(f, V)=1$.

Proof. Let $M=\bigcap_{n \geq 1} \mathrm{cl}\left(f^{n}(E)\right)$. Then since $\gamma\left(\operatorname{cl}\left(f^{n}(E)\right)\right) \leq k^{n} \gamma(E)$, it is easy to check (using Proposition 1 ) that $M$ is attractor for compact sets under $f$. Corollary 7 now follows from Corollary 6. Q.E.D.

Corollary 8. (See [24] or [25].) Let $G$ be a closed, convex subset of a Banach space $X$ and $f: G \rightarrow G$ a k-set-contraction, $k<1$. Assume that there exists an integer $N$ such that $f^{N}(G)$ is bounded. Then $f$ bas a fixed point.

Proof. Let $E_{0}=f^{N}(G)$ and $E_{j}=f^{j}\left(E_{0}\right)$ for $1 \leq j \leq N-1$. Then if we define $E=\bigcup_{j=0}^{N-1} E_{j}, E$ is a bounded set, $f(E) \subset E$, and the other hypothes is of Corollary 7 holds. Q.E.D.

Corollary 8 is a direct generalization of Darbo's theorem (Proposition 3). However, the condition that $f^{N}(G)$ be bounded for an unbounded $G$ is usually too strong to be of use in applications.

At this point we would like to recall a long-standing conjecture in fixed point theory.

Conjecture. Let $G$ be a closed, bounded convex set in a Banach space and $f: G \rightarrow G$ a continuous map. Assume that there exists an integer $N \geq 1$ such that $f^{N}$ is compact. Then $f$ has a fixed point (?).

We should remark that A. and V. Istratescu have claimed this result in [35], but their proof appears incomplete.

We wish to prove the above conjecture (as a special case) with the additional unaesthetic assumption that $f$ restricted to an appropriate open set is continuously Fréchet differentiable. This suggests that the conjecture is true, but our line of argument breaks down in the general case.

The spirit of our proof will be as follows: If $f$ in the conjecture is a linear map, then $f$ respects convexity properties and one can easily show that $K_{\infty}(f, G)$ is compact and $f\left(K_{\infty}(f, G)\right) \subset K_{\infty}(f, G)$, so that $f$ has a fixed point. If $f$ is nonlinear, but continuously Fréchet differentiable, locally $f$ is almost linear. We shall show below that by careful exploitation of this local linearity we can still show that $f$ has a fixed point in the general case, though the argument is necessarily more involved.

Before stating our result we shall need a number of lemmas. Lemma 10 below was first proved in [28]; a proof is also given in [29, p. 191]. 
Lemma 10 ([28], [29]). Let $V$ be an open subset of a Banach space $X$ and $g$ : $V \rightarrow X$ a k-set-contraction. Assume that $g$ is Fréchet differentiable at $x \in V$. Then $d g_{x}$, the Fréchet derivative of $g$ at $x$, is a k-set-contraction.

Lemma 11. Let $M$ be a compact subset of a Banach space $X, U$ an open neighborbood of $M$, and $f: U \rightarrow X$ a continuously Fréchet differentiable map. Then given $\epsilon>0$ and a positive integer $n$, there exists $\delta>0$ such that

$$
\left\|d f_{x_{n}} d f_{x_{n-1}} \cdots d f_{x_{1}}-d f_{y_{n}} d f_{y_{n-1}} \cdots d f_{y_{1}}\right\|<\epsilon
$$

for all points $x_{1}, x_{2}, \cdots, x_{n}$ and $y_{1}, y_{2}, \cdots, y_{n} \in M$ such that $\left\|x_{i}-y_{i}\right\|<\delta$ for $1 \leq i \leq n$.

Proof. The map $\left(x_{1}, x_{2}, \cdots, x_{n}\right) \rightarrow d f_{x_{1}} d f_{x_{2}} \cdots d f_{x_{n}}$ is a continuous map from $\Pi_{i=1}^{n} M=Y=a$ compact set to $L(X, X)$. Since $Y$ is compact, the map is uniformly continuous. Q.E.D.

Before proceeding further we need to establish some temporary notation. Let $C_{1}, C_{2}, \cdots, C_{p}$ be closed, convex subsets of a Banach space $X$ and $f: C=$ $\bigcup_{i=1}^{p} C_{i} \rightarrow X$ a map. For any subset $A$ of $X$ define $b_{i}(A)=\operatorname{co} f\left(A \cap C_{i}\right)$, define $A_{1}=\bigcup_{i=1}^{p} b_{i}(A)$ and generally define $A_{m+1}=\bigcup_{i=1}^{p} b_{i}\left(A_{m}\right)$. For each $j, 1 \leq j \leq$ $p$, define

$$
I(j)=\left\{i: 1 \leq i \leq p, \overline{\mathrm{CO}} f\left(C_{i}\right) \cap C_{j} \neq \varnothing\right\} .
$$

If $J=\left(i_{1}, i_{2}, \cdots, i_{m}\right)$ is an $m$-tuple of integers $1 \leq i_{j} \leq p$, such that $i_{j-1} \in I\left(i_{j}\right)$ for $2 \leq j \leq m$, we shall say that $J$ is a "permissible $m$-tuple of integers"; and if $i_{1}=i$, we shall say that $J$ is a "permissible $m$-tuple whose first entry is $i$." This notation provides a slightly more precise description of the set $A_{2}$ corresponding to a set $A \subset C$, namely

$$
A_{2}=\bigcup_{i_{2}=1}^{p} b_{i_{2}}\left(A_{1}\right)=\bigcup_{i_{2}=1}^{p} b_{i_{2}}\left(\bigcup_{i_{1} \in I\left(i_{2}\right)} b_{i_{1}}(A)\right) .
$$

Of course all of the notation given here depends on the particular covering $\left\{C_{i}\right\}$ and the map $f$, but in the following these should be obvious from the context so we will not mention them explicitly.

Lemma 12. Let $M$ be a compact subset of a Banach space $X, U$ an open neighborbood of $M$ and $f: U \rightarrow X$ a continuously Fréchet differentiable map such that $f(M) \subset M$. Let $\epsilon>0$ be a given positive number and $n$ a positive integer. Then there exists a number $r>0$ such that if $\left\{V_{s}\left(x_{i}\right): 1 \leq i \leq p, x_{i} \in M\right\}$ is any finite closed covering of $M$ by balls of radius $s \leq r$ and if $J=\left(i_{1}, i_{2}, \cdots, i_{n}\right)$ any permissible n-tuple of integers (with respect to this covering), then

$$
\left\|\left(d f_{x_{i_{n}}} d f_{x_{i_{n-1}}} \cdots d f_{x_{i_{1}}}\right)-d\left(f^{n}\right)_{x_{i_{1}}}\right\|<\epsilon .
$$


Proof. Given $\epsilon>0$ and $n$, let $\delta>0$ be as in Lemma 11. By a compactness and continuity argument, we can assume that $\left\|d f_{x}\right\| \leq K$ for $x \in U_{1}, U_{1}$ an open neighborhood of $M$ contained in $U$. Take $r>0$ such that if $\rho=(K+1)^{n} r, \rho<\delta$ and $N_{\rho}(M)=\{x: d(x, M)<\rho\} \subset U_{1}$. Let $\left\{V_{s}\left(x_{i}\right): 1 \leq i \leq p\right\}$ be a closed covering of $M$ by balls of radius $s, s \leq r$, and let $J=\left(i_{1}, i_{2}, \cdots, i_{n}\right)$ be a permissible $n$-tuple with respect to this covering. For notational convenience define $z_{1}=x_{i_{1}}$ and $z_{j}=f^{j-1}\left(z_{1}\right)$ for $2 \leq j \leq n$. It follows from the chain rule that $d\left(f^{n}\right) x_{i_{1}}=$ $d f_{z_{n}} d f_{z_{n-1}} \cdots d f_{z_{1}}$. Thus, using Lemma 11, it suffices to show that $\left\|x_{i_{j}}-z_{j}\right\| \leq$ $(K+1)^{j}$ for $1 \leq j \leq n$. This is obviously true for $j=1$. Assume we have proved for some $j \geq 1$ that $\left\|x_{i_{j}}-z_{j}\right\| \leq(K+1)^{j} r$. By our assumptions, the ball of radius $(K+1)^{j} r$ about $z_{j}$ is contained in $U_{1}$ and $f$ is Lipschitz with constant $K$ on this ball.

$$
\therefore\left\|f\left(x_{i_{j}}\right)-f\left(z_{j}\right)\right\|=\left\|f\left(x_{i_{j}}\right)-z_{j+1}\right\| \leq K(K+1)^{j} r .
$$

By the definition of permissible $m$-tuple $\overline{\text { co }} f\left(V_{s}\left(x_{i_{j}}\right)\right) \cap V_{s}\left(x_{i_{j+1}}\right) \neq \varnothing$, and since $f \mid V_{s}\left(x_{i_{j}}\right)$ is a Lipschitz map with constant $K, \overline{\operatorname{co}} f\left(V_{s}\left(x_{i_{j}}\right)\right) \subset V_{K s}\left(f\left(x_{i_{j}}\right)\right)$. It follows that $\left\|x_{i_{j+1}}-f\left(x_{i}\right)\right\| \leq(K+1) r$. Therefore we have that $\left\|x_{i_{j+1}}-z_{j+1}\right\| \leq$ $(K+1) r+K(K+1)^{j} r \leq(K+1)^{j+1} r$. Q.E.D.

Lemma 13. Let $M$ be a compact subset of a Banach space $X, U$ an open neighborbood of $M$ and $f: U \rightarrow X$ a continuously Fréchet differentiable map such that $f(M) \subset M$. Assume that for some fixed integer $n, f^{n}$ (which is defined on an open neigbborbood of $M$ ) is a k-set-contraction. Then if $c>k$, there exists a number $r>0$ such that if $\left\{V_{s}\left(x_{i}\right): 1 \leq i \leq p, x_{i} \in M\right\}$ is any finite closed covering of $M$ by balls of radius $s \leq r$ and if $J=\left(i_{1}, i_{2}, \cdots, i_{n}\right)$ any permissible $n$-tuple of integers, then $\left(d f_{x_{i_{n}}} d f_{x_{i_{n-1}}} \cdots d f_{x_{i_{1}}}\right)$ is a $1 / 2(k+c)$-set-contraction.

Proof. In the notation of Lemma 12, take $\epsilon=1 / 2(c-k)$ and let $r$ be the number guaranteed by Lemma 12. Then

$$
\left\|\left(d f_{x_{i_{n}}} d f_{x_{i_{n-1}}} \cdots d f_{x_{i_{1}}}\right)-d\left(f^{n}\right)_{x_{i_{1}}}\right\| \leq 1 / 2(c-k) .
$$

But by Lemma $10 d\left(f^{n}\right)_{x_{i}}$ is a $k$-set-contraction. Q.E.D.

We are now in a position to establish our main lemma.

Lemma 14. Let $M$ be a compact subset of a Banach space $X, U$ an open neighborbood of $M$, and $f: U \rightarrow X$ a continuously Fréchet differentiable map sucb that $f(M) \subset M$. Assume that for some fixed integer $n, f^{n}$ (which is defined on an open neighborbood of $M$ ) is a k-set-contraction, $k<1$. Select $c$ sucb that $k<c<1$. Then there exists $r>0$ such that if $\left\{V_{s}\left(x_{i}\right): 1 \leq i \leq p, x_{i} \in M\right\}$ is any closed covering of $M$ by balls of radius $s, s \leq r$, and if $A$ is any subset of $V=\bigcup_{i=1}^{p} V_{s}\left(x_{i}\right)$, 
$\gamma\left(A_{n}\right) \leq c \gamma(A)$, where $A_{j}$ is taken with respect to $\left\{V_{s}\left(x_{i}\right)\right\}$.

Proof. Given $x$ in the domain of $f$, let $d f_{x}$ denote the Fréchet derivative of $f$ at $x$ and $R_{x}$ the remainder at $x$, so if $y$ is near $x, f(y)=f(x)+d f_{x}(y-x)+R_{x}(y)$, where $R_{x}(y)=o(\|y-x\|)$. By a continuity and compactness argument we can assume that $\left\|d f_{x}\right\| \leq K$ for $x \in U_{1}=$ an open neighborhood of $M$. Select $r_{1}>0$ such that if $\rho=(K+1)^{n} r_{1}, N_{\rho}(M) \subset U_{1}$. Let $\epsilon>0$ be a number such that $n \epsilon K^{n}<$ $1 / 2(c-k)$. By another compactness and continuity argument there exists $r_{2}>0$ such that for any $x \in M, R_{x} \mid V_{r_{2}}(x)$ is a Lipschitz map with Lipschitz constant less than $\epsilon$. Let $r_{3}>0$ be the number whose existence is guaranteed by Lemma 13. Let $r=\min \left\{r_{1}, r_{2}, r_{3}\right\}$ and let $\left\{V_{s}\left(x_{i}\right): 1 \leq i \leq p, x_{i} \in M\right\}$ be a closed covering of $M$ by balls of radius $s, s \leq r$.

For notational convenience we define $V_{i}=V_{s}\left(x_{i}\right), L_{i}=d f_{x_{i}}, R_{i}=R_{x_{i}}$ and $T_{i}$ by $T_{i}(y)=f\left(x_{i}\right)+L_{i}\left(y-x_{i}\right)$. If $J=\left(i_{1}, i_{2}, \cdots, i_{m}\right)$ is any $m$-tuple of integers with $1 \leq i_{j} \leq p$, we define $L_{j}=L_{i_{m}} L_{i_{m-1}} \cdots L_{i_{1}}$ and $T_{J}=T_{i_{m}} T_{i_{m-1}} \cdots T_{i_{1}}$. By Lemma $13, L_{J}$ is a $1 / 2(k+c)$-set-contraction if $J$ is a permissible $n$-tuple of integers with respect to $\left\{V_{s}\left(x_{i}\right)\right\}$; and consequently $T_{J}$ (which is just a translate of $L_{J}$ ) is also a $1 / 2(k+c)$-set-contraction.

Now let $A$ be any subset of $V=\bigcup_{i=1}^{p} V_{s}\left(x_{i}\right)$. We can assume that $A \cap V_{i}$ is convex for $1 \leq i \leq p$. Otherwise simply replace $A$ by $A^{\prime}=\bigcup_{i=1}^{p} \operatorname{co}\left(V_{i} \cap A\right)$ and note that $\gamma\left(A^{\prime}\right)=\gamma(A)$. As a first step in estimating $\gamma\left(A_{m}\right)$ for $1 \leq m \leq n$, notice that $f \mid V_{i}$ is a Lipschitz map of constant $K$, so that $f \mid V$ is a $K$-set-contraction. It follows easily that $\gamma\left(A_{m}\right) \leq K^{m} \gamma(A)$.

Our strategy from here on is to use finite induction to "approximate" the sets $A_{m}, 1 \leq m \leq n$. First, observe that

$$
\begin{aligned}
b_{i}(A) & =\operatorname{co}\left(T_{i}+R_{i}\right)\left(A \cap V_{i}\right) \subset \operatorname{co}\left[T_{i}\left(A \cap V_{i}\right)+R_{i}\left(A \cap V_{\mathrm{i}}\right)\right] \\
& \subset T_{i}\left(A \cap V_{i}\right)+\operatorname{co}\left(\bigcup_{j=1}^{p} R_{j}\left(A \cap V_{j}\right)\right)=T_{i}\left(A \cap V_{i}\right)+\theta_{1}(A),
\end{aligned}
$$

where $\theta_{1}(A)=\operatorname{co}\left(\bigcup_{j=1}^{p} R_{j}\left(A \cap V_{j}\right)\right)$. We see that $\gamma\left(\theta_{1}(A)\right) \leq \epsilon \gamma(A)$. Generally, suppose for some $m, 1 \leq m \leq n$, we have shown that for $1 \leq i \leq p, b_{i}\left(A_{m-1}\right) \subset$ $\operatorname{co}\left\{T_{J}\left(A \cap V_{i_{1}}\right): J\right.$ varies over all permissible $m$-tuples whose first entry is $i_{1}$ and whose $m$ th entry is $i\}+\theta_{m}(A)$, where $\theta_{m}(A)$ denotes a convex set such that $\gamma\left(\theta_{m}(A)\right) \leq m \in K^{m-1} \gamma(A)$. (In this induction $A_{0}$ is defined to be $A$. ) We then have that

$$
b_{i}\left(A_{m}\right)=b_{i}\left(\bigcup_{j \in I(i)} b_{j}\left(A_{m-1}\right)\right) \subset \cot T_{i}\left(\bigcup_{j \in I(i)} b_{j}\left(A_{m-1}\right)\right)+\operatorname{co}\left(\bigcup_{j=1}^{p} R_{j}\left(A_{m} \cap V_{j}\right)\right) .
$$


Using the inductive hypothesis on the form of $b_{j}\left(A_{m-1}\right)$ and the fact $T_{i}$ respects convex structure (for any set $S, T_{i}(\cos S)=\cot T_{i}(S)$ ), it follows that co $T_{i}\left(\bigcup_{j \in I(i)} b_{j}\left(A_{m-1}\right)\right) \subset \operatorname{co}\left\{T_{J^{\prime}}\left(A \cap V_{i_{1}}\right): J^{\prime}\right.$ ranges over all permissible $(m+1)$-tuples whose first entry is $i_{1}$ and whose $(m+1)$ th entry is $\left.i\right\}+$ $\operatorname{co}\left(\bigcup_{j=1}^{p} T_{j}\left(\theta_{m}(A)\right)\right)$. Thus, if we define

$$
\theta_{m+1}(A)=\operatorname{co}\left(\bigcup_{j=1}^{p} R_{j}\left(A_{m} \cap V_{j}\right)\right)+\operatorname{co}\left(\bigcup_{j=1}^{p} T_{j}\left(\theta_{m}(A)\right)\right),
$$

to check the inductive step it suffices to show that $\gamma\left(\theta_{m+1}(A)\right) \leq$ $(m+1) \epsilon K^{m+1} \gamma(A)$. But by inductive hypothesis, $\gamma\left(\theta_{m}(A)\right) \leq m \epsilon K^{m} \gamma(A)$, so using the properties of the measure of noncompactness we find that

$$
\gamma\left(\operatorname{co}\left(\bigcup_{j=1}^{p} T_{j}\left(\theta_{m}(A)\right)\right)\right) \leq K \gamma\left(\theta_{m}(A)\right) \leq m \epsilon K^{m+1} \gamma(A) .
$$

On the other hand, $R_{j} \mid V_{j}$ is an $\epsilon$-set-contraction and $\gamma\left(A_{m} \cap V_{j}\right) \leq K^{m} \gamma(A)$, so

$$
\gamma\left(\operatorname{co}\left(\bigcup_{j=1}^{p} R_{j}\left(A_{m} \cap V_{j}\right)\right)\right) \leq \epsilon K^{m} \gamma(A) .
$$

Putting these facts together we see that $\gamma\left(\theta_{m+1}(A)\right) \leq(m+1) \epsilon K^{m+1} \gamma(A)$ (assuming, as we can, that $K \geq 1$ ).

This completes the inductive step, and since $A_{n}=\bigcup_{i=1}^{p} b_{i}\left(A_{n-1}\right)$, it follows easily that

$$
A_{n} \subset \operatorname{co}\left\{T_{J}(A): J \text { a permissible } n \text {-tuple }\right\}+\theta_{n}(A),
$$

where $\gamma\left(\theta_{n}(A)\right) \leq n \epsilon K^{n} \gamma(A) \leq 1 / 2(c-k) \gamma(A)$. However, by our selection of $r$ and by Lemma 13, $\gamma\left(T_{J}(A)\right) \leq 1 / 2(c+k) \gamma(A)$, for any permissible $n$-tuple $J$. Putting these facts together we find that

$$
\gamma\left(A_{n}\right) \leq \max \left\{\gamma\left(T_{J}(A)\right): J \text { a permissible } n \text {-tuple }\right\}+\gamma\left(\theta_{n}(A)\right) \leq c \gamma(A) . \quad \text { Q.E.D. }
$$

Corollary 9. Let $G$ be a closed, convex subset of a Banacb space $X$ and f: $G \rightarrow G$ a continuous map. Assume that $\stackrel{\circ}{G}$, the interior of $G$, is nonempty and that there exists a set $M \subset G$ which is an attractor for compact sets under f. Finally, assume that there exists an open neigbborbood $V$ of $M$ and an integer $n \geq 1$ sucb that $f^{n} \mid V$ is a k-set-contraction, $k<1$, and $f \mid V$ is continuously Fréchet differentiable. Then $f$ has a fixed point.

Proof. Select $c$ such that $k<c<1$ and let $r$ be as in Lemma 14. We can also assume that $N_{r}(M) \subset V$. Let $\left\{x_{i}: 1 \leq i \leq p\right\}$ be a $1 / 2 r$-net of points in $M$ and let $S=$ $\bigcup_{i=1}^{p} V_{r}\left(x_{i}\right)$, so that $S$ contains an open neighborhood of $M$. By Lemma 5 there exists a bounded open neighborhood $W$ of $M, W \subset V$, such that $f(W) \subset W$. Since $f^{j}(W)$ 
is a decreasing sequence of sets and since $\gamma\left(f^{j n}(W)\right) \leq k^{j} \gamma(W), \lim _{j \rightarrow \infty} \gamma\left(f^{j}(W)\right)=$ 0 . By Lemma 9 there exists a bounded open neighborhood $U$ of $M$ such that $U \subset$ interior $(S)$ and $\mathrm{cl} f(U) \subset U$.

We now define a decreasing sequence of sets $\left\{B_{j}\right\}$ by

$$
B_{1}=\bigcup_{i=1}^{p} \bar{c} \bar{o} f\left(U \cap V_{i}\right)
$$

and

$$
B_{j}=\bigcup_{i=1}^{p} \overline{c o} f\left(U \cap B_{j-1} \cap V_{i}\right) \text { for } j>1, V_{i}=V_{r}\left(x_{i}\right) .
$$

It is easy to see that $B_{j} \supset B_{j+1}$ for $j \geq 1$ and that $f\left(U \cap B_{j}\right) \subset B_{j+1}$ for $j \geq 1$. It follows that if we set $B_{\infty}=\bigcap_{j \geq 1} B_{j}, B_{\infty}$ is also a union of $p$ closed, convex sets and $f\left(U \cap B_{\infty}\right) \subset B_{\infty}$ or (since $\left.\mathrm{cl}^{\prime} f(U) \subset U\right) \mathrm{cl} f\left(U \cap B_{\infty}\right) \subset U \cap B_{\infty}$. If we define

$$
A_{1}=\bigcup_{i=1}^{p} \overline{\mathrm{co}} f\left(V_{i}\right)
$$

and

$$
A_{j}=\bigcup_{i=1}^{p} \overline{\mathrm{co}} f\left(V_{i} \cap A_{j-1}\right) \text { for } j>1,
$$

it is easy to see that $A_{j} \supset B_{j}$ for $j \geq 1$. Also, it follows by Lemma 14 (since the measure of noncompactness is not affected by taking closures) that $\gamma\left(A_{j n}\right) \leq$ $c^{j} \gamma(S)$, and this implies that $\lim _{j \rightarrow \infty} \gamma\left(B_{j}\right)=0$. By Proposition $1, B_{\infty}$ is compact and nonempty and $B_{j}$ approaches $B_{\infty}$ in the Hausdorff metric.

The rest of our proof simply mimics that of Corollary 6 . Just as before there exists $\delta>0$ and an integer $j_{2}$ such that $N_{2 \delta}\left(f\left(U \cap B_{j}\right)\right) \cap B_{j} \subset U \cap B_{j}$ and $\gamma\left(f\left(U \cap B_{j}\right)\right)<2 \delta$ for $j \geq j_{2}$. For $j \geq j_{2}$ let $f\left(U \cap B_{j}\right)=\bigcup_{i=1}^{m(j)} S_{i, j}$, where diameter $\left(S_{i, j}\right)<2 \delta$. If we define $U_{i, j}=\overline{\mathrm{co}} S_{i, j} \cap B_{j}$ for $j \geq j_{2}$ and $U_{j}=\bigcup_{i=1}^{m(j)} U_{i, j}$, it is clear from the construction that $U_{j} \in \mathcal{F}_{0}$ and that $f\left(U_{j}\right) \subset U_{j}$ (since $f\left(U \cap B_{j}\right) \subset U_{j} \subset$ $\left.U \cap B_{j}\right)$. If we define $M_{1}=M \cup \operatorname{cl}\left(B_{\infty} \cap U\right)$, then $M_{1}$ is an attractor for compact sets under $f$, and it is easy to check that the hypotheses of Theorem 1 hold with $M_{1}$ and $\left\{U_{j}\right\}$. Therefore $f$ has a fixed point. Q.E.D.

We could obtain more general consequences of Corollary 9, but we would like to restrict ourselves to the following simple result:

Corollary 10. Let $G$ be a closed, bounded convex set with nonempty interior $\stackrel{\circ}{G}$ and $f: G \rightarrow G$ a continuous map. Assume that there exists an integer $n$ such that $f^{n}$ is a k-set-contraction, $k<1$, (this will be true if $f^{n}$ is compact) and suppose that $M=\bigcap_{j} \geq 1 \mathrm{cl}\left(f^{j}(G)\right) \subset \stackrel{\circ}{G}$. Assume that there exists an open neighborbood $V$ of $M$ such that $f \mid V$ is continuously Fréchet differentiable. Then $f$ bas a fixed point. 
Proof. By Corollary 9 it suffices to show that $M$ is an attractor for compact sets under $f$. However, since $\gamma\left(\operatorname{cl}\left(f^{n j}(G)\right)\right) \leq k^{j} \gamma(G)$, it follows that $\lim _{j \rightarrow \infty} \gamma\left(f^{j}(G)\right)=0$. Therefore, by Proposition $1, \mathrm{cl}\left(f^{j}(G)\right)$ approaches $M$ in the Hausdorff metric, so $M$ is certainly an attractor for compact sets. Q.E.D.

Unfortunately the aforementioned conjecture in its stated generality remains as untractable as ever.

3. Our goal in this section is to show that some of our previous results actually hold for spaces more general than closed convex subsets of a Banach space. Our main interest is in obtaining fixed point theorems for functions defined on open subsets of closed convex sets, but with essentially the same amount of work we can obtain more general theorems. We then specialize to the cases of interest. We begin by extracting a part of the proof of Theorem 1 and isolating it as a lemma:

Lemma 15. Suppose that in a Banach space $X$ there exists a sequence of closed, nonempty sets $\left\{U_{n}: n \geq 1\right\}$, a sequence of positive real numbers $\left\{r_{n}: n \geq 1\right\}$, a compact set $M$, and a continuous function fefined on $\bigcup_{n=1}^{\infty} U_{n}$ such that the following bold: (1) $U_{n} \in \mathcal{F}_{0}$ for $n \geq 1$ (i.e., eacb $U_{n}$ is a finite union of closed, convex sets) and $f\left(U_{n}\right) \subset U_{n}$ for $n \geq 1$. (2) $\Lambda\left(f \mid U_{n}\right)$, the Lefschetz number of $f \mid U_{n}$, is nonzero for $n \geq 1$ (it follows from Lemma 1 that each $U_{n}$ bas the same bomology as a compact, metric ANR, so the ordinary Lefschetz number is defined).

(3) $\lim _{n \rightarrow \infty} r_{n}=0$ and $U_{n} \subset N_{r_{n}}(M)$. Then $f$ bas a fixed point.

As we have remarked, Lemma 15 is essentially contained in the proof of Theorem 1 .

Our strategy for obtaining fixed point theorems from here on will be to use Lemma 15 to replace Theorem 1 and to make further assumptions to guarantee that $\Lambda\left(f \mid U_{n}\right) \neq 0$, a fact which previously followed automatically. We need one more lemma.

Lemma 16. Suppose that $G$ is a closed subset of a Banach space $X$ and that $G \in \mathcal{F}$ (i.e., $G$ has a locally finite cover by closed, convex sets). Suppose that $K$ is a compact subset of $G, K \in \mathcal{F}_{0}$ and $K$ is contractible in itself to a point. Then given any $\epsilon>0$ there exists a set $K(\epsilon) \subset G$ such that $K(\epsilon) \in \mathcal{F}_{0}, K(\epsilon) \subset N_{\epsilon}(K)=$ $\{x \in G: d(x, K) \leq \epsilon\}, K(\epsilon)$ contains an open neighborbood in $G$ of $K$ and $K(\epsilon)$ is contractible in itself to a point.

Proof. Suppose that $G=\bigcup_{i \in I} G_{i}$, where $\left\{G_{i}\right\}$ is a locally finite covering of $G$ by closed, convex sets. Since $K$ is compact, there exists an open neighborhood $U$ of $K$ in $G$ and a finite set of indices $F \subset I$ such that $U \cap G_{i}=\varnothing$ for $i \notin F$. Since $K \in \mathcal{F}_{0}, K=\bigcup_{j=1}^{n} C_{j}$, where the $C_{j}$ are closed and convex. If we define $K_{i, j}=$ $G_{i} \cap C_{j}$ for $i \in F$ and $1 \leq j \leq n$ we see that $K=\bigcup_{(i, j)} K_{i, j^{*}}$

Now suppose that $\epsilon>0$. Take $\epsilon_{0}, 0<\epsilon_{0}<\epsilon$, so small that $N_{\epsilon_{0}}(K) \subset U$. Given $\delta>0$, define $K_{i, j}(\delta)=\left\{x \in G_{i}: d\left(x, K_{i, j}\right) \leq \delta\right\}$. It is easy to see that there exists 
$\epsilon_{1}, 0<\epsilon_{1}<\epsilon_{0}$, such that for any $J \subset\{(i, j): i \in F, 1 \leq j \leq n\}, \bigcap_{(i, j) \epsilon J} K_{i, j}\left(\epsilon_{1}\right)$ $\neq \varnothing$ if and only if $\bigcap_{(i, j) \in J} K_{i, j} \neq \varnothing$. Take $\epsilon_{2}=\min \left\{\epsilon_{0}, \epsilon_{1}\right\}$ and define $K(\epsilon)=$ $\bigcup_{(i, j)} K_{i, j}\left(\epsilon_{2}\right)$. Obviously $K(\epsilon)$ is contained in $U$, and by Lemma $1 K$ (which is contractible in itself to a point) is a deformation retraction of $K(\epsilon)$, so $K(\epsilon)$ is contractible in itself to a point.

It remains to show that $K(\epsilon)$ contains an open neighborhood in $G$ of $K$. Suppose not, so there exists a sequence of points $x_{n} \in G$ such that $d\left(x_{n}, K\right) \rightarrow 0$ but $x_{n} \notin K(\epsilon)$. By taking a subsequence we can assume that $\left\{x_{n}\right\} \subset G_{i}$ for some fixed $i$, and by taking a further subsequence we can assume that $x_{n} \rightarrow x \in K_{i, j}$ for some $j, 1 \leq j \leq n$. It follows that $x_{n} \in K_{i, j}\left(\epsilon_{2}\right)$ for $n$ large enough, contradicting the original assumption. Q.E.D.

We now want to generalize Frum-Ketkov's theorem. Basically the proof parallels that of Corollary 1.

Theorem 2. Suppose that $G \in \mathcal{F}, U$ is an open subset of $G$ and $f: U \rightarrow U$ is a continuous map. Assume that: (1) There exists a compact set $M \subset U$ which is an

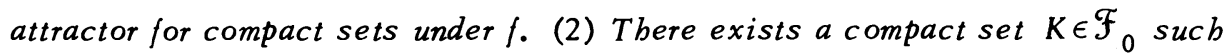
that $M \subset K \subset U$ and such that $K$ is contractible in itself to a point. (This followed automatically before.) (3) There exists a real-valued function $\rho:[0, a] \rightarrow[0, a]$ $(a>0)$ such that $\rho$ is continuous from the right, $\rho(r)<r$ for $0<r \leq a$, and $f\left(N_{r}(M)\right)$ $C N_{\rho(r)}(M)$ for $0 \leq r \leq a$. Then $f$ has a fixed point.

Proof. Let $\left\{a_{k}\right\}$ be a sequence of positive numbers approaching 0 and let $b_{k}=\rho\left(a_{k}\right)$ for $k \geq 1$. (We can assume that $a_{k} \leq a$ and that if $\left\{G_{i}: i \in I\right\}$ is the locally finite covering of $G$ by closed, convex sets, $N_{a}(M) \cap G_{i}$ is empty except for finitely many $i_{\text {.) }}$ Just as in Corollary 1 , if we let $\left\{x_{i, k}: 1 \leq i \leq n(k)\right\}$ be a $1 / 2\left(a_{k}-b_{k}\right)$-net of points on $M, V_{i, k}=\left\{x \in G:\left\|x-x_{i, k}\right\| \leq a_{k}\right\}$ and $U_{k}=$ $\bigcup_{i=1}^{n(k)} V_{i, k}$ then $U_{k} \in \mathcal{F}_{0}$ and $f\left(U_{k}\right) \subset U_{k}$. Thus to prove that $f$ has a fixed point, it suffices by Lemma 15 to show that $\Lambda\left(f \mid U_{k}\right)=1$ for all $k$.

Since $M$ is an attractor for compact sets, there exists an integer $n_{0}$ such that $f^{n} 0(K) \subset N_{b_{k}}(M) \subset \stackrel{\circ}{U}_{k}$. If $K(\epsilon)$ is as in Lemma 16 , there exists $\epsilon$ so small that $f^{n} 0(K(\epsilon)) \subset U_{k}$. If we define $V_{k}=U_{k} \cup \bigcup_{j=0}^{n_{0}} f^{j}(K(\epsilon))$ it is clear that $f\left(V_{k}\right) \subset V_{k}$ and in fact $f^{n} 0\left(V_{k}\right) \subset U_{k}$. It follows by the properties of the generalized Lefschetz number that $\Lambda_{\text {gen }}\left(f \mid V_{k}\right)=\Lambda_{g \text { en }}\left(f \mid U_{k}\right)$, so it suffices to show $\Lambda_{g e n}\left(f \mid V_{k}\right)=1$. The properties of $\rho$ imply that $\lim _{n \rightarrow \infty} \rho^{n}\left(a_{k}\right)=0$, so hypothes is (3) of the theorem implies that given any open neighborhood $W$ of $M$, there exists an integer $n_{1}$ such that $f^{n}\left(U_{k}\right) \subset W$ for $n \geq n_{1}$. But by Lemma $16, K(\epsilon)$ contains an open neighborhood (in $G$ ) of $M$, so there exists an integer $n_{1}$ with $f^{n}\left(U_{k}\right) \subset K(\epsilon)$ for $n \geq n_{1}$ and hence $f^{n}\left(V_{k}\right) \subset K(\epsilon)$ for $n \geq n_{1}+n_{0}$. By Lemma 4 (since $K(\epsilon)$ is contractible in itself to a point), $\Lambda_{\mathrm{gen}}\left(f \mid V_{k}\right)=1$. Q.E.D.

Remark. Notice that it was not necessary to know that $M$ be an attractor for 
all compact sets, only that it be an attractor for $K$.

We can obtain a number of consequences of Theorem 2. A more or less typical result is the following corollary, which has similarities to Theorem 6 of Horn's article [16].

Corollary 11. Let $G$ be a closed, convex subset of a Banach space, $\Omega$ an open subset of $G, G_{0}$ an open, convex subset of $\Omega$ and $f: \Omega \rightarrow G$ a continuous map. Assume that $\mathrm{cl}\left(f^{j}\left(G_{0}\right)\right) \subset \Omega$ for $1 \leq j \leq 2 n-1$ and that $\mathrm{cl}\left(f^{j}\left(G_{0}\right)\right) \subset G_{0}$ for $n \leq j \leq$ $2 n-1$. Suppose that there exists a set $M \subset G_{0}$ which is an attractor for compact subsets of $G_{0}$ (i.e. $M$ is compact, $f(M) \subset M$, and given any compact set $A \subset G_{0}$ and any open neighborbood $W$ of $M, f^{j}(A) \subset W$ for $j$ large enougb). Finally assume that for $0 \leq r \leq a, a>0, f\left(N_{r}(M)\right) \subset N_{\rho(r)}(M)$, where $\rho$ is a function as in Theorem 2 . Then $f$ has a fixed point.

Proof. Suppose we can prove there exists an open subset $U$ of $\Omega$ such that $G_{0} \subset U, f(U) \subset U$ and $f^{2 n-1}(U) \subset G_{0}$. It would then follow that $M$ is an attractor for compact subsets of $U$ under $f$, and if (in the notation of Theorem 2) we defined $K=\overline{c o}(M), f$ would have a fixed point (by Theorem 2 ).

It remains to construct $U$. Let $A_{j}=\mathrm{cl}\left(f^{j}\left(G_{0}\right)\right)$ for $1 \leq j \leq 2 n-1$. Since $A_{2 n-1}$ is a closed subset of $G_{0}$, there exists an open neighborhood $G_{2 n-1}$ of $A_{2 n-1}$ such that $\mathrm{cl}\left(G_{2 n-1}\right) \subset G_{0}$. Since cl $f\left(A_{2 n-2}\right) \subset G_{2 n-1}$, there exists an open neighborhood $G_{2 n-2}$ of $A_{2 n-2}$ such that cl $\left(f\left(G_{2 n-2}\right)\right) \subset G_{2 n-1}$ and $\operatorname{cl}\left(G_{2 n-2}\right) \subset G_{0}$ if $2 n-2 \geq n$. Continuing in this way we obtain open neighborhoods $G_{j}$ of $A_{j}$ for $1 \leq j \leq 2 n-1$ such that $\operatorname{cl}\left(f\left(G_{j}\right)\right) \subset G_{j+1}$ for $1 \leq j \leq 2 n-1$ $\left(G_{2 n}\right.$ is defined to be $\left.G_{0}\right)$ and such that $\mathrm{cl}\left(G_{j}\right) \subset G_{0}$ for $n \leq j \leq 2 n-1$. If we define $U=\bigcup_{j=0}^{2 n-1} G_{j}$, it is not hard to show that $\operatorname{cl}(f(U)) \subset U$ and $\operatorname{cl}\left(f^{2 n-1}(U)\right) \subset$ $G_{0}$. Q.E.D.

We want to show next that Corollary 6 also has a generalization for spaces more general than closed, convex sets. Aside from some technical changes, the proof closely parallels that of Corollary 6 , so we shall be sketchy in the presentation.

Theorem 3. Suppose that $G \in \mathcal{F}, W$ is an open subset of $G$ and $f: W \rightarrow W$ is a continuous map. Assume that there exists a compact set $M \subset W$ which is an attractor for compact subsets of W under f. Suppose that there exists an open neighborbood $V$ of $M$ such that $f \mid V$ is a k-set-contraction, $k<1$. Finally, suppose that there exists a compact set $K \in \mathcal{F}_{0}$ such that $M \subset K \subset W$ and $K$ is contractible in itself to a point. Then $i_{G}(f, V)=1$ and $f$ bas a fixed point.

Proof. Just as in Corollary 6 there exists a bounded, open neighborhood $U$ in $G$ of $M$ such that $\mathrm{cl}(f(U)) \subset U$. If $\left\{G_{i}: i \in I\right\}$ is a locally finite cover of $G$ by closed, convex sets we can also assume that $G_{i} \cap U$ is empty except for $i \in F$, a finite subset of $I$. If we define $K_{n}^{*}=K_{n}(f, U) \cap G$ and $K_{\infty}^{*}=K_{\infty}(f, U) \cap G$, then 
again as in Corollary 6 there exists an integer $n_{2}$ such that $N_{2 \delta}\left(f\left(U \cap K_{n}^{*}\right)\right) \cap$ $K_{n}^{*} \subset U \cap K_{n}^{*}$ and $\gamma\left(f\left(U \cap K_{n}^{*}\right)\right)<2 \delta$ for $n \geq n_{2}$. For $n \geq n_{2}$, let $f\left(U \cap K_{n}^{*}\right)=$ $\bigcup_{j=1}^{m(n)} S_{j, n}$, where diameter $S_{j, n}<\delta$, set $U_{j, n}=\overline{\operatorname{co}}\left(S_{j, n}\right) \cap G$ and define $U_{n}=$ $\bigcup_{j} U_{j, n^{*}}$ It is clear from the construction that $U_{n} \in \mathcal{F}_{0}$ (since each $U_{j, n}$ is a finite union of closed, convex sets) and that $f\left(U_{n}\right) \subset U_{n}$.

To show that $f$ has a fixed point it suffices by Lemma 15 to show that $\Lambda\left(f \mid U_{n}\right)=1$. Since $M$ is an attractor for compact sets there exists an integer $N$ such that $f^{j}(K) \subset U$ for $j \geq N$. In the notation of Lemma 12 take $\epsilon$ so small that $f^{N}(K(\epsilon)) \subset U$. Since $f^{j}(U) \subset U_{n}$ for $j \geq n+1$, it follows that $f^{j}(K(\epsilon)) \subset U_{n}$ for $j \geq$ $N+n+1$. Thus if we define $V_{n}=U_{n} \cup \bigcup_{j=0}^{N+n+1} f^{j}(K(\epsilon)), f\left(V_{n}\right) \subset V_{n}$ and $f^{j}\left(V_{n}\right) \subset$ $U_{n}$ for $j \geq N+n+1$. As previously this implies that $\Lambda_{g \text { en }}\left(f \mid V_{n}\right)=\Lambda_{g \text { en }}\left(f \mid U_{n}\right)$. However, $K(\epsilon)$ contains an open neighborhood of $M$, so by Lemma 9 there exists an integer $N_{1}$ such that $f^{j}(U) \subset K(\epsilon)$ for $j \geq N_{1}$. It follows that for $j \geq N+N_{1}$, $f^{j}\left(V_{n}\right) \subset K(\epsilon)$, so Lemma 4 implies that $\Lambda_{g \text { en }}\left(f \mid V_{n}\right)=1$, and $f$ has a fixed point.

The proof that $i_{G}(f, V)=1$ closely parallels the proof of the same fact in Corollary 6 , and we leave it to the reader. We only note that some slight modifications are necessary since $G$ is now a locally finite union of closed, convex sets instead of a closed, convex set. Q.E.D.

Theorem 3 is closely related to Theorem 1 of [24] or [25], but it does not seem directly comparable.

As an easy consequence of Theorem 3 we obtain the following result, which could also be derived from Theorem 1 in [25]:

Corollary 12. Let $G$ be a closed, convex subset of a Banacb space, $\Omega$ an open subset of $G, G_{0}$ a bounded open convex subset of $\Omega$ and $f: \Omega \rightarrow G$ a k-set-contraction, $k<1$. Assume that $\mathrm{cl}\left(G_{0}\right) \subset \Omega$ and that $\mathrm{cl}\left(f^{j}\left(G_{0}\right)\right) \subset \Omega$ for $1 \leq j \leq 2 n-1$ ( $n$ some fixed integer) and $\mathrm{cl}\left(f^{j}\left(G_{0}\right)\right) \subset G_{0}$ for $n \leq j \leq 2 n-1$. Then $i_{G}\left(f, G_{0}\right)$ is defined, $i_{G}\left(f, G_{0}\right)=1$, and $f$ has a fixed point.

Proof. If $S$ is the set of fixed points of $f$ in $\mathrm{cl}\left(G_{0}\right), S=f^{n}(S) \subset \mathrm{cl}\left(f^{n}\left(G_{0}\right)\right) \subset$ $G_{0}$, so $S$ is a closed subset of $G_{0}$. Also since $f(S)=S, \gamma(S)=\gamma(f(S)) \leq k \gamma(S)$, and we must have $\gamma(S)=0$, so $S$ is compact. Thus $i_{G}\left(f, G_{0}\right)$ is defined.

By the same argument as in Corollary 11, there exists a bounded open subset $W$ of $\Omega$ such that $G_{0} \subset W, \operatorname{cl}(f(W)) \subset W$ and $\operatorname{cl}\left(f^{2 n-1}(W)\right) \subset G_{0}$. Let $A_{j}=\operatorname{cl}\left(f^{j}(W)\right)$ and $A_{\infty}=M=\bigcap_{j \geq 1} A_{j}$. Then by Proposition $1, M$ is an attractor for compact sets under $f$, and by construction, $M \subset G_{0}$. If we define $K=\overline{c o}(M)$, the corollary now follows from Theorem 3. Q.E.D.

Corollary 10 of $\$ 2$ also admits some generalizations. We mention one of these, but we omit the proof, since it involves no new ideas:

Corollary 13. Let $\Omega$ be a bounded open subset of a Banach space $X, G_{0}$ a bounded open convex subset of $\Omega$, and $f: \Omega \rightarrow X$ a continuous map. Assume that 
for some integer $n, \mathrm{cl}\left(f^{j}\left(G_{0}\right)\right) \subset \Omega$ for $0 \leq j \leq 2 n-1$ and $\mathrm{cl}\left(f^{j}\left(G_{0}\right)\right) \subset G_{0}$ for $n \leq$ $j \leq 2 n-1$. Assume that $f \mid G_{0}$ is continuously Fréchet differentiable and that for some integer $m, f^{m} \mid G_{0}$ is a k-set-contraction, $k<1$. Then $f$ has a fixed point.

4. The results of the previous sections are not meant to be definitive, and we would like to mention some open questions and conjectures which are suggested by our previous theorems. Our first conjecture was suggested to the author by F. E. Browder several years ago.

Conjecture. Let $G$ be a closed, convex subset of a Banach space $X$ and $f$ : $G \rightarrow G$ a continuous map. Assume that there exists a set $M \subset G$ which is an attractor for compact sets under $f$ (see $\$ 1$ for the definition). Then $f$ has a fixed point (?).

If this conjecture were true, it would easily imply all the results of $\$ \S 1$ and 2 -including the long-standing question raised in $\$ 2$. We suspect the conjecture as stated is false, but we cannot give a counterexample. None of the well-known counterexamples of fixed point theory satisfy the above hypotheses. As a means of establishing the bounds of the theory, it would be very interesting to find a counterexample to the conjecture-and it would be even more interesting to prove it (if it is true).

The theorems of $\$ 3$ raise a natural question: Since $G \in \mathcal{F}$ implies that $G$ is a metric ANR, do the theorems of $\$ 3$ (or analogous results) hold for metric ANR's? It is not hard to show that the condition that $G \in \mathcal{F}$ can at least be somewhat weakened. To get results like Theorems 2 and 3, it suffices that $G$ be a complete metric space and that for each $c>1$ there exist an isometric imbedding $j_{c}$ of $G$ into $G_{c} \in \mathcal{F}$ and a retraction $r_{c}$ of some open neighborhood $U_{c}$ of $j_{c}(G)$ onto $j_{c}(G)$ such that $r_{c}$ is a $c$-set-contraction. The unit sphere in a Banach space can be shown to satisfy this condition. However, we can see no reason why a general completely metrizable ANR should possess a family of imbeddings as above. As a final conjecture we raise the following question:

Conjecture. Let $G$ be a closed, bounded convex subset of a Banach space $X$ and $f: G \rightarrow X$ a continuous map such that $f(\partial G) \subset G$. Assume that there exists a compact set $M \subset X$ and a constant $c<1$ such that $d(f(x), M) \leq c d(x, M)$ for all $x \in G$. Then $f$ has a fixed point (?).

The above conjecture (for $G$ a ball) is actually the theorem which Frum-Ketkov claimed to prove in [13]. If $X$ is essentially a $\Pi_{1}$-space and $G$ is a ball the result is proved in [25], and a slight improvement of the method used there establishes it for general closed, bounded, convex sets (with nonempty interior) in a $\Pi_{1}$-space. This conjecture is the sort of result one usually proves with the aid of degree theory, and in fact one can define a degree theory for maps of the form $I$ $f$, where $f$ satisfies a "Frum-Ketkov condition." The trouble is that the class of such maps is not at all nice. For instance, suppose $G$ is a closed, bounded, convex 
subset of a Banach space $X$. Assume that $f_{i}(i=0,1): G \rightarrow X$ and suppose there exists a constant $c<1$ and a compact set $M_{i}$ such that $d\left(f_{i}(x), M_{i}\right) \leq c d\left(x, M_{i}\right)$ for all $x \in G$. If for $0 \leq t \leq 1$ we define $f_{t}(x)=(1-t) f_{0}+t f(x)$, it is by no means clear that there exists a compact set $M_{t}$ such that $d\left(f_{t}(x), M_{t}\right) \leq c d\left(x, M_{t}\right)$ for all $x \in G$.

\section{REFERENCES}

1. Ju. G. Borisovič and Ju. I. Sapronov, A contribution to the topological theory of condensing operators, Dokl. Akad. Nauk SSSR 183 (1968), 18-20 = Soviet Math. Dokl. 9 (1968), 1304-1307. MR 38 \#6414.

2. F. E. Browder, On a generalization of the Schauder fixed point theorem, Duke Math. J. 26 (1959), 291-303. MR 21 \#4368.

3. - On the fixed point index for continuous mappings of locally connected spaces, Summa Brasil. Math. 4 (1960), 253-293. MR 26 \#4354.

4. - Another generalization of the Schauder fixed point theorem, Duke Math. J. 32 (1965), 399-406. MR $34 \# 3567$.

5. - A further generalization of the Schauder fixed point theorem, Duke Math. J. 32 (1965), 575-578. MR $34 \# 3568$. $43 \# 1165$.

6. —, Asymptotic fixed point theorems, Math. Ann. 185 (1970), 38-60. MR

7. - Nonlinear operators and nonlinear equations of evolution, Proc. Sympos.

Pure Math., vol. 18, part 2, Amer. Math. Soc., Providence, R.I. (to appear).

8. G. Darbo, Punti uniti in trasformazioni a codominio non compatto, Rend. Sem.

Mat. Univ. Padova 24 (1955), 84-92. MR 16, 1140.

9. A. Deleanu, Théorie des points fixes: sur les rétractes de voisinage des espaces convexoïdes, Bull. Soc. Math. France 87 (1959), 235-243. MR 26 \#763.

10. A. Dold, Fixed point index and fixed point theorem for Euclidean neighborhood retracts, Topology 4 (1965), 1-8. MR 33 \#1850.

11. R. Driver, Existence and continuous dependence of solutions of a neutral functional-differential equation, Arch. Rational Mech. Anal. 19 (1965), 149-166. MR 31 \#3654.

12. J. Dugundji, An extension of Tietze's theorem, Pacific J. Math. 1 (1951), 353367. MR 13, 373.

13. R. L. Frum-Ketkov, Mappings into a Banach space sphere, Dokl. Akad. Nauk SSSR 175 (1967), 1229-1231 = Soviet Math. Dok1. 8 (1967), 1004-1007. MR 36 \#3181.

14. J. K. Hale, A class of neutral equations with the fixed point property, Proc. Nat. Acad. Sci. U.S.A. 67 (1970), 136-137.

15. - Existence, uniqueness and continuous dependence for hereditary systems, Ann. Mat. Pura Appl. (4) 85 (1970), 63-81. MR 41 \#7238.

16. W. A. Horn, Some fixed point theorems for compact maps and flows in Banach spaces, Trans. Amer. Math. Soc. 149 (1970), 391-404. MR 42 \#2334.

17. G. S. Jones, Asymptotic fixed point theorems and periodic systems of functionaldifferential equations, Contributions to Differential Equations 2 (1963), 385-405. MR $28 \# 1361$.

18. - Stability and asymptotic fixed-point theory, Proc. Nat. Acad. Sci. U.S.A. 53 (1965), 1262-1264. MR 31 \#4959.

19. M. A. Krasnosel 'ski1, The operator of translation along the trajectories of differential equations, "Nauka", Moscow, 1966; English transl., Transl. Math. Monographs, vol. 19, Amer. Math. Soc., Providence, R. I., 1968. MR 34 \#3012; MR 36 \#6688.

20. C. Kuratowski, Sur les espaces complets, Fund. Math. 15 (1930), 301-309.

21. J. Leray, Sur la position d'un ensemble fermé de points d'un espace topologique, J. Math. Pures Appl. (9) 24 (1945), 169-199. MR 7, 468.

22. - Sur les équations et les transformations, J. Math. Pures Appl. (9) 24 (1946), 201-248. MR 7, 468. 
23. J. Leray, Théorie des points fixes: indice total et nombre de Lefschetz, Bull. Soc. Math. France 87 (1959), 221-233. MR 26 \#762.

24. R. D. Nussbaum, The fixed point index and asymptotic fixed point theorems for $k$-set-contractions, Bull. Amer. Math. Soc. 75 (1969), 490-495. MR 39 \#7589.

25. - Asymptotic fixed point theorems for local condensing maps, Math. Ann. 191 (1971), $181-195$.

26. The fixed point index for local condensing maps, Ann. Mat. Pura Appl. 89 (1971), 217-258.

27. - A geometric approach to the fixed point index, Pacific J. Math. 39 (1971), $751-766$.

28. - The fixed point and fixed point theorems for $k$-set-contractions, Ph. D. Dissertation, University of Chicago, Chicago, Ill., 1969 (unpublished).

29. - Estimates for the number of solutions of operator equations, Applicable Analysis 1 (1971), 183-200.

30. B. O'Neill, Essential sets and fixed points, Amer. J. Math. 75 (1953), 497-509. MR 15, 145.

31. B. N. Sadovskił, On a fixed point principle, Funkcional. Anal. i Priložen. 1 (1967), no. 2, 74-76. (Russian) MR 35 \#2184.

32. H. Steinlein, Zur existenz von fixpunkten bei abbildungen mit vollstetigen iterierten, Ph. D. Dissertation, University of Munich, 1970.

33. R. B. Thompson, A unified approach to local and global fixed point indices, Advances in Math. 3 (1969), 1-71. MR 40 \#891.

34. T. Yoshizawa, Stable sets and periodic solutions in a perturbed system, Contributions to Differential Equations 2 (1963), 407-420. MR 28 \#1362.

35. A. Istratescu and V. Istratescu, On the theory of fixed points for some classes of mappings. II, Rev. Roumaine Math. Pures Appl. 16 (1971), 1073-1076.

DEPARTMENT OF MATHEMATICS, RUTGERS UNIVERSITY, NEW BRUNSWICK, NEW JERSEY 08903 